\title{
DISCOVERY OF TWO NEARBY PECULIAR L DWARFS FROM THE 2MASS PROPER-MOTION SURVEY: YOUNG OR METAL-RICH? ${ }^{1}$
}

\author{
Dagny L. Looper, ${ }^{2,3}$ J. Davy Kirkpatrick, ${ }^{3,4}$ Roc M. Cutri, ${ }^{4}$ Travis Barman, ${ }^{5}$ Adam J. Burgasser,${ }^{3,6}$ \\ Michael C. Cushing, ${ }^{2,7}$ Thomas Roellig, ${ }^{8}$ Mark R. McGovern, ${ }^{9}$ Ian S. Mclean, ${ }^{10}$ \\ Emily Rice, ${ }^{10}$ Brandon J. Swift, ${ }^{7}$ and Steven D. Schurr ${ }^{4}$ \\ Received 2008 May 5; accepted 2008 June 4
}

\begin{abstract}
We present the discovery of two nearby L dwarfs from our 2MASS proper-motion search, which uses multiepoch 2MASS observations covering $\sim 4700 \mathrm{deg}^{2}$ of sky. 2MASS J18212815+1414010 and 2MASS J21481628+4003593 were overlooked by earlier surveys due to their faint optical magnitudes and their proximity to the Galactic plane $\left(10^{\circ} \leq|b| \leq 15^{\circ}\right)$. Assuming that both dwarfs are single, we derive spectrophotometric distances of $\sim 10 \mathrm{pc}$, thus increasing the number of known L dwarfs within 10 pc to 10. In the near-infrared, 2MASS J21481628+4003593 shows a triangular $H$-band spectrum, strong $\mathrm{CO}$ absorption, and a markedly red $J-K_{S}$ color $(2.38 \pm 0.06)$ for its L6 optical spectral type. 2MASS J18212815+1414010 also shows a triangular $H$-band spectrum and a slightly red $J-K_{s}$ color $(1.78 \pm 0.05)$ for its L4.5 optical spectral type. Both objects show strong silicate absorption at $9-11 \mu \mathrm{m}$. Cumulatively, these features imply an unusually dusty photosphere for both of these objects. We examine several scenarios to explain the underlying cause for their enhanced dust content and find that a metal-rich atmosphere or a low surface gravity are consistent with these results. 2MASS J18212815+1414010 may be young (and therefore have a low surface gravity) based on its low tangential velocity of $10 \mathrm{~km} \mathrm{~s}^{-1}$. On the other hand, 2MASS J21481628+4003593 has a high tangential velocity of $62 \mathrm{~km} \mathrm{~s}^{-1}$ and is therefore likely old. Hence, high metallicity and low surface gravity may lead to similar effects.
\end{abstract}

Subject headings: stars: individual (2MASS J18212815+1414010, 2MASS J21481628+4003593) stars: low-mass, brown dwarfs — techniques: spectroscopic

Online material: color figure

\section{INTRODUCTION}

Many of the objects known to reside in the solar neighborhood were discovered via their high proper motion. Early propermotion surveys by the pioneers of photographic astrometry built the foundation of our knowledge about the local stellar census (e.g., Luyten 1979, 1980; Giclas et al. 1978; Ross 1939; Wolf 1918; Barnard 1916; Innes 1915). Discoveries of our nearest stellar and substellar neighbors continue to date, with surveys such as the SIPS survey (Deacon \& Hambly 2007; Deacon et al. 2005), the LEHPM survey (Pokorny et al. 2004), the SCR sur-

\footnotetext{
1 Based in part on data collected at Subaru Telescope, which is operated by the National Astronomical Observatory of Japan. Some of the data presented herein were obtained at the W. M. Keck Observatory, which is operated as a scientific partnership among the California Institute of Technology, the University of California, and the National Aeronautics and Space Administration. The Observatory was made possible by the generous financial support of the W. M. Keck Foundation.

${ }^{2}$ Institute for Astronomy, University of Hawaii, 2680 Woodlawn Drive, Honolulu, HI 96822.

3 Visiting Astronomer at the Infrared Telescope Facility, which is operated by the University of Hawaii under Cooperative Agreement NCC 5-538 with the National Aeronautics and Space Administration, Office of Space Science, Planetary Astronomy Program.

4 Infrared Processing and Analysis Center, Mail Stop 100-22, California Institute of Technology, Pasadena, CA 91125.

5 Lowell Observatory, 1400 West Mars Hill Road, Flagstaff, AZ 86001.

6 MIT Kavli Institute for Astrophysics and Space Research, 77 Massachusetts Avenue, Building 37-664B, Cambridge, MA 02139.

7 Steward Observatory, University of Arizona, 933 North Cherry Avenue, Tucson, AZ 85721

8 NASA Ames Research Center, Moffett Field, CA 94035-1000.

9 Antelope Valley College, 3041 West Avenue K, Lancaster, CA 93536.

10 Department of Physics and Astronomy, University of California, Los Angeles, CA 90095-1562.
}

vey (Finch et al. 2007), and the SUPERBLINK survey (Lépine et al. 2002, 2008; Lépine 2008). Despite their successes, these surveys are not ideally suited to the discovery of nearby brown dwarfs because of their dependence on optical data. As we have now learned from longer wavelength surveys such as the Two Micron All Sky Survey (2MASS; Skrutskie et al. 2006), the Deep Near Infrared Survey of the southern sky (DENIS; Epchtein et al. 1997), and the Sloan Digital Sky Survey (SDSS; York et al. 2000), the solar neighborhood is home to many L and T dwarfsthe coldest brown dwarfs currently known (Kirkpatrick 2005). Most of these cool objects have been selected photometrically using color selections appropriate for $\mathrm{L}$ and $\mathrm{T}$ dwarfs with near-solar metallicity. This method may be missing low-temperature objects with unusual physical properties - low- or high-metallicity dwarfs or objects with unusual atmospheric traits-but a measurement of their proper motions will help to identify them as nearby.

Another benefit of proper-motion surveys over photometric ones is their ability to find nearby objects against the confusion of the Galactic plane. Due to reddening caused by intervening material, extinguished background objects in the plane can have colors mimicking those of nearby dwarfs in 2MASS, DENIS, and SDSS colors. Proper motions help ferret out these nearby interlopers without relying on color information.

Using data contained in the 2MASS Survey Working Databases, we have searched for proper-motion objects by examining the subset of multiepoch data having time differences in excess of 2 months. The total coverage satisfying this criterion is approximately $4700 \mathrm{deg}^{2}$, or $\sim 11 \%$ of the sky. In this paper we present two discoveries from this exclusively near-infrared search. Both objects are optically faint, nearby L dwarfs located within $15^{\circ}$ of the Galactic plane and hence missed by previous optical proper-motion 
TABLE 1

2Mass Measurements for the Two New L Dwarfs

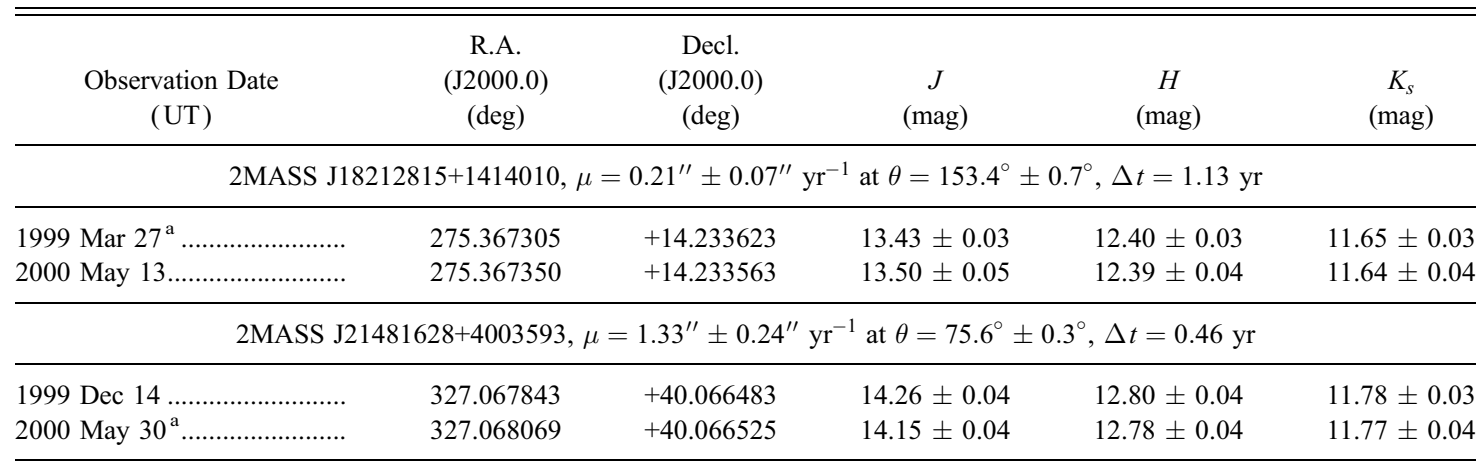

${ }^{\text {a }}$ Entries in the 2MASS Point Source Catalog.

and near-infrared photometric surveys. We briefly describe our 2MASS proper-motion search and measurements from the 2MASS catalog in $\S 2$, along with the observations; we give the analysis in $\S 3$, discussion in $\S 4$, and conclusions in $\S 5$.

\section{OBSERVATIONS}

\subsection{Discovery}

We have conducted a near-infrared (NIR) proper-motion search using the 2MASS database for high proper-motion and late-type, optically faint objects (Burgasser et al. 2007; Looper et al. 2007b). 2MASS was designed to be a single-epoch survey covering the entire sky, but for various quality control or scientific reasons, multiple regions of the sky were observed more than once, totaling $\sim 4700 \mathrm{deg}^{2}$ of sky observed over at least two epochs. We briefly describe our search criteria here; a complete description of the survey will be presented in a forthcoming publication (J. D. Kirkpatrick et al. 2009, in preparation).

Candidates were required to have $J \leq 15.8$, no optical counterparts ( $B$ and $R$ bands) within $5^{\prime \prime}$ in the USNO-B1 Catalog (Monet et al. 2003), positional differences of $0.4^{\prime \prime} \leq \Delta x \leq 1.5^{\prime \prime}$ between the first and last epochs, first- and last-epoch time differences of $\Delta t \geq 0.2 \mathrm{yr}$, proper motions of $\mu \geq 0.2^{\prime \prime} \mathrm{yr}^{-1}$, and to be located at least $10^{\circ}$ away from the Galactic plane. After this initial query, candidates were examined visually using charts constructed from 2MASS $J, H$, and $K_{s}$ bands; Digitized Sky Survey (DSS) I $R, B$, or $V$ band; and DSS II $R$ band. DSS I and II images were obtained from the CADC service. ${ }^{11}$ Those without visible counterparts in the DSS I or II images or, for particularly bright objects, with large $R-J$ colors $(R-J \gtrsim 6)$, were selected for follow-up spectroscopy. Here we present two noteworthy objects selected for early follow-up based on their bright 2MASS magnitudes.

\subsection{Two Bright L Dwarf Candidates}

The first candidate has a 2MASS All-Sky Point Source Catalog designation of 2MASS J18212815+1414010, hereafter abbreviated as 2MASS J1821+1414. Astrometric and photometric measurements from the two separate epochs listed in the 2MASS Survey Point Source Working Database are given in Table 1. Despite this object having bright $J$-band $(13.43 \pm 0.03)$ and $K_{s}$-band (11.65 \pm 0.03$)$ magnitudes, it is invisible in the DSS I $B$-band and DSS II $R$-band plates. It is, however, visible in the DSS II $I$-band plate (see Fig. 1). Its large implied optical-toinfrared color, along with a $J-K_{s}$ color of $1.78 \pm 0.04$, suggests

11 See http://cadcwww.dao.nrc.ca/cadcbin/getdss. that it is a mid- to late-type $L$ dwarf. Using the two available 2MASS epochs and a positional error estimate of $0.06^{\prime \prime}$, we calculate a proper motion of $\mu=0.21^{\prime \prime} \pm 0.07^{\prime \prime}$ at P.A. $=153.4^{\circ} \pm 0.7^{\circ}$.

The second candidate has a designation of 2MASS J21481628+ 4003593, hereafter abbreviated as 2MASS J2148+4003. Measurements of this object from the 2MASS Survey Point Source Working Database are also given in Table 1.2MASS J2148+4003 has a very red $J-K_{s}$ color $(2.38 \pm 0.06)$ and bright $J$-band $(14.15 \pm 0.04)$ and $K_{s}$-band $(11.77 \pm 0.04)$ magnitudes and is invisible in the DSS I $R$-band and DSS II $R$-band plates. It is visible on the DSS II $I$-band plate (see Fig. 1). Using the two available 2MASS epochs and a positional error estimate of $0.06^{\prime \prime}$, we calculate a high proper motion of $\mu=1.33^{\prime \prime} \pm 0.24^{\prime \prime}$ at P.A. $=$ $75.6^{\circ} \pm 0.3^{\circ}$. We show the position of both 2MASS J1821+1414 and 2MASS J2148+4003 on a NIR color-color diagram, along with the average colors of main-sequence dwarfs and $\mathrm{L}$ and $\mathrm{T}$ dwarfs with 2MASS photometry in Figure 2. Both objects lie along the redward extension of late-type $\mathrm{M}$ to $\mathrm{L}$ dwarfs.

In the following sections we describe observations of 2MASS $1821+1414$ and 2MASS J2148+4003 carried out using SubaruFOCAS, Keck-NIRSPEC, IRTF-SpeX, and Spitzer-IRS, along with data reductions. The summary for all observations can be found in Table 2.

\subsection{Subaru-FOCAS Spectroscopy}

The optical spectroscopic observations were carried out on 2007 August 20 and 21 (UT) at the Subaru Telescope on Mauna Kea, Hawaii, using the Faint Object Camera and Spectrograph (FOCAS; Kashikawa et al. 2002). FOCAS was used with the 300R grating blazed at $7500 \AA$ and the S058 filter to block second-order light from wavelengths shorter than $5800 \AA$. The grating tilt was set so that the wavelength region from 5850 to $10250 \AA$ was covered. Use of a long slit of width $0.8^{\prime \prime}$ resulted in a resolution of $8.5 \AA$. Both nights were clear, but with variable seeing. On 2007 August 20 the seeing varied from $0.5^{\prime \prime}$ to $2.5^{\prime \prime}$. On 2007 August 21 the seeing was somewhat more stable, ranging only from $0.6^{\prime \prime}$ to $1.5^{\prime \prime}$. Subaru employs an atmospheric dispersion corrector, so keeping the slit aligned with the parallactic angle was not necessary.

The data were reduced and calibrated using standard IRAF ${ }^{12}$ routines. The overscan region of the array was used for bias subtraction, and a median of five dome flats taken on the first night was used to normalize the response of the detector. Because of

12 IRAF is distributed by the National Optical Astronomy Observatory, which is operated by the Association of Universities for Research in Astronomy, Inc., under cooperative agreement with the National Science Foundation. 

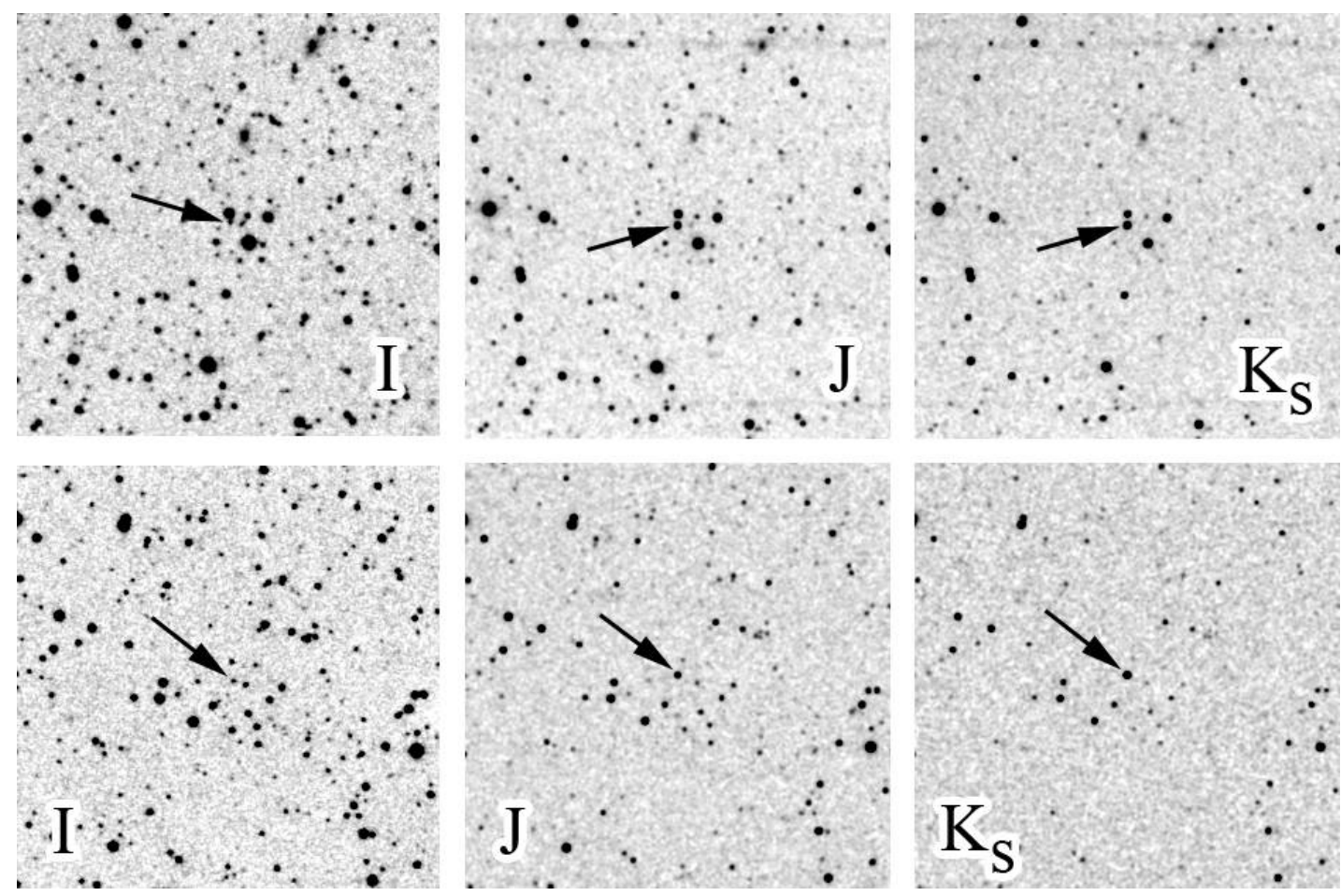

FIG. 1. - Finder charts for 2MASS J18212815+1414010 (top row) and 2MASS J21481628+4003593 (bottom row). Each field is centered on the target and is $5^{\prime}$ wide with north up and east to the left. From left to right, the three images for 2MASS J1821+1414 are $I$ band from the DSS II (epoch 1993 June 29), $J$ band from 2MASS (epoch 1999 March 27), and $K_{s}$ band from 2MASS (epoch 1999 March 27). For 2MASS J2148+4003 the images are $I$ band from DSS II (epoch 1993 September 22), $J$ band from 2MASS (epoch 2000 May 30), and $K_{s}$ band from 2MASS (epoch 2000 May 30). Arrows mark the location of the objects on the DSS II and 2MASS images.

strong, broad spectral signatures in the flat-field lamps themselves, special care was needed during the flat-field correction step. The FOCAS chip is designed to be used with multiobject spectroscopy, but for our single-slit observations most of the detector area was not used or needed for sky subtraction. This allowed us to perform a block average (in the spatial dimension) on a much smaller $21 \times 3260$ pixel region of the median dome flat corresponding to the area where the primary target spectrum

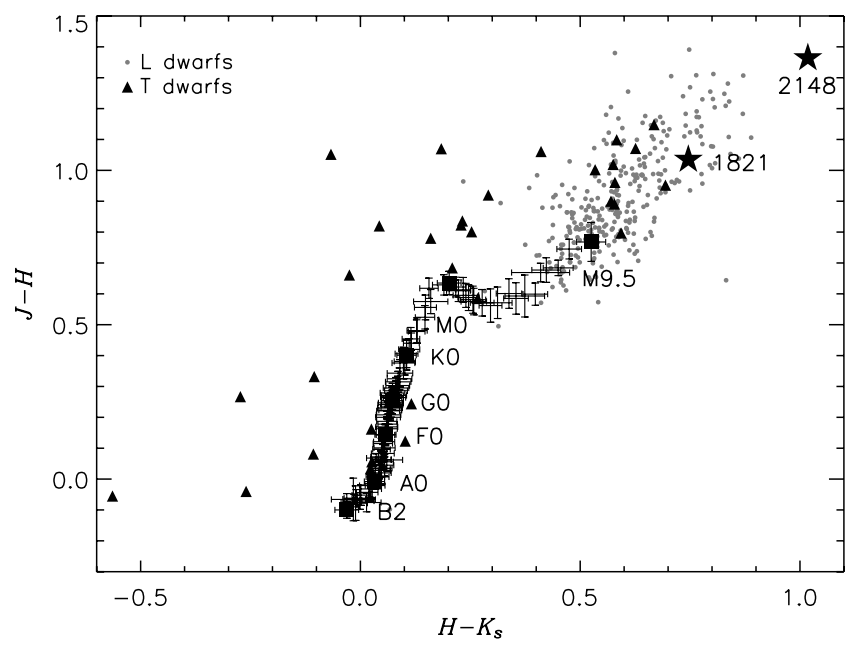

Fig. 2.- Near-infrared color-color plot $\left(H-K_{s}\right.$ vs. $\left.J-H\right)$ showing (1) average colors of B2-M9.5 dwarfs in the 2MASS Point Source Catalog, with each half-subtype represented by a point and its associated error bar and average colors for labeled spectral types (black squares); (2) L dwarfs with 2MASS photometry (see http://dwarfarchives.org) and $\sigma_{J-H}$ and $\sigma_{H-K_{s}} \leq 0.1$ (gray circles); (3) T dwarfs with 2MASS photometry and $\sigma_{J-H}$ and $\sigma_{H-K_{s}} \leq 0.2$ (triangles); and (4) the positions of 2MASS J1821+1414 and 2MASS J2148+4003 ( five-pointed stars). [See the electronic edition of the Journal for a color version of this figure.] and sky subtraction region would fall in subsequent exposures. This block-averaged, one-dimensional, vertical slice encompasses the gross undulations of the dome flat in the spectral dimension, and we replicated this slice across the spatial dimension to create a two-dimensional image. This image was then divided back into the median flat to produce a map that contains only small-scale flat-field variations. This map was then normalized using IMSURFIT and divided into all other data frames to remove the flatfield signature across the detector.

The individual stellar spectra were then extracted using the APALL package. Wavelength calibration was achieved using arc lamps taken after each program object. Spectra were flux-calibrated using an observation of LTT 9491 (Hamuy et al. 1994) taken on the first night. We checked this fluxing using another standard, Wolf 1346 (Massey \& Gronwall 1990), taken on the second night, and the agreement was excellent. Dwarfs of type G0 were also acquired after each program target, and these were used to correct for telluric absorption in the program objects. Specifically, the G0 dwarf SAO 10356 was used for 2MASS J1821+1414 and the G0 dwarf SAO 207827 was used for 2 MASS J2148+4003. The telluric bands removed by this method are those of $\mathrm{O}_{2}$ at 6867-7000 (the Fraunhofer B band) and 7594-7685 $\AA$ (the Fraunhofer A band), as well as $\mathrm{H}_{2} \mathrm{O}$ at $7186-7273,8162-8282$, and 8950-9650 $\mathrm{A}$.

\subsection{Keck-NIRSPEC Spectroscopy}

2MASS J1821+1414 and 2MASS J2148+4003 were observed with the Near-Infrared Spectrometer (NIRSPEC; McLean et al. $1998,2000)$ on the $10 \mathrm{~m} \mathrm{~W}$. M. Keck Observatory. Using a $1024 \times 1024 \mathrm{InSb}$ array and the spectrograph in low-resolution mode, we selected the N3 filter to cover the part of the $J$-band window from 1.14 to $1.36 \mu \mathrm{m}$, where the most diagnostic NIR features lie. Use of the $0.38^{\prime \prime}$ slit results in a resolving power of $R \equiv \lambda / \Delta \lambda \approx 2500$. For the observations of 2 MASS J1821+1414 
TABLE 2

Observation Log

\begin{tabular}{|c|c|c|c|c|c|c|c|}
\hline Object $^{\mathrm{a}}$ & Telescope/Instrument & $\begin{array}{c}\lambda \\
(\mu \mathrm{m})\end{array}$ & $\lambda / \Delta \lambda$ & UT Date & $z$ & $\begin{array}{c}N \times t \\
(\mathrm{~s})^{\mathrm{b}}\end{array}$ & Calibrator \\
\hline 2MASS J02052940-1159296................. & Subaru/FOCAS & $0.585-1.025$ & 1000 & 2007 Aug 20 & 1.2 & $2 \times 1200$ & . \\
\hline 2MASS J18212815+1414010 …….......... & Subaru/FOCAS & $0.585-1.025$ & 1000 & 2007 Aug 20 & 1.0 & $2 \times 1200$ & SAO 10356 (G0 V) \\
\hline 2MASS J18212815+1414010................... & Keck/NIRSPEC & $1.14-1.36$ & 2500 & $2005 \mathrm{Jul} 18$ & 1.0 & $4 \times 300$ & HD 165029 (A0 V) \\
\hline 2MASS J18212815+1414010................... & IRTF/SpeX & $0.7-2.5$ & 100 & 2005 Aug 10 & 1.0 & $8 \times 120$ & HD 165029 (A0 V) \\
\hline 2MASS J18212815+1414010_.................. & IRTF/SpeX & $0.9-2.4$ & 1200 & 2005 Aug 10 & 1.1 & $12 \times 300$ & HD 165029 (A0 V) \\
\hline 2MASS J18212815+1414010 …............... & IRTF/SpeX & $1.9-4.2$ & 1500 & 2007 Jul 28 & 1.5 & $40 \times 30$ & HD 165029 (A0 V) \\
\hline 2MASS J18212815+1414010 …….......... & Spitzer/IRS & $5.2-15.3$ & 90 & AOR 16201984 & $\ldots$ & 3904 & \\
\hline 2MASS J21321145+1341584................... & Subaru/FOCAS & $0.585-1.025$ & 1000 & 2007 Aug 21 & 1.2 & $2 \times 1200$ & \\
\hline 2MASS J21481628+4003593.................. & Subaru/FOCAS & $0.585-1.025$ & 1000 & 2007 Aug 20 & 1.2 & $3 \times 1200$ & SAO 207827 (G0 V) \\
\hline 2MASS J21481628+4003593.................. & Subaru/FOCAS & $0.585-1.025$ & 1000 & 2007 Aug 21 & 1.1 & $2 \times 1200$ & SAO 207827 (G0 V) \\
\hline 2MASS J21481628+4003593.................. & Keck/NIRSPEC & $1.14-1.36$ & 2500 & 2005 Dec 9 & 1.2 & $4 \times 300$ & SAO 71693 (A0 V) \\
\hline 2MASS J21481628+4003593................... & Keck/NIRSPEC & $0.94-1.15$ & 2500 & 2006 Aug 4 & 1.2 & $4 \times 200$ & HD $207220(A 0 \mathrm{~V})$ \\
\hline 2MASS J21481628+4003593.................... & IRTF/SpeX & $1.9-4.2$ & 1500 & 2006 Sep 1 & 1.1 & $40 \times 30$ & HD 209932 (A0 V) \\
\hline 2MASS J21481628+4003593................... & IRTF/SpeX & $1.9-4.2$ & 1500 & 2006 Sep 12 & 1.1 & $40 \times 30$ & HD 209932 (A0 V) \\
\hline 2MASS J21481628+4003593................... & IRTF/SpeX & $1.9-4.2$ & 1500 & 2006 Sep 12 & 1.1 & $40 \times 30$ & HD 209932 (A0 V) \\
\hline 2MASS J21481628+4003593.................... & IRTF/SpeX & $1.9-4.2$ & 1500 & 2006 Sep 12 & 1.1 & $40 \times 30$ & HD 209932 (A0 V) \\
\hline 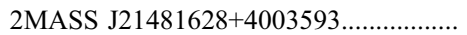 & IRTF/SpeX & $1.9-4.2$ & 1500 & 2007 Nov 11 & 1.1 & $40 \times 30$ & HD 13936 (A0 V) \\
\hline 2MASS J21481628+4003593................... & IRTF/SpeX & $1.9-4.2$ & 1500 & 2007 Nov 11 & 1.1 & $40 \times 30$ & HD 13936 (A0 V) \\
\hline 2MASS J21481628+4003593................... & IRTF/SpeX & $1.9-4.2$ & 1500 & 2007 Nov 11 & 1.2 & $40 \times 30$ & HD 13936 (A0 V) \\
\hline 2MASS J21481628+4003593................... & IRTF/SpeX & $1.9-4.2$ & 1500 & 2007 Nov 11 & 1.2 & $40 \times 30$ & HD 13936 (A0 V) \\
\hline 2MASS J21481628+4003593................... & IRTF/SpeX & $1.9-4.2$ & 1500 & 2007 Nov 11 & 1.3 & $40 \times 30$ & HD 209932 (A0 V) \\
\hline 2MASS J21481628+4003593................... & Spitzer/IRS & $5.2-15.3$ & 90 & AOR 16176128 & $\ldots$ & 3904 & \\
\hline 2MASS J22443167+2043433................... & IRTF/SpeX & $0.7-2.5$ & 100 & 2005 Sep 9 & 1.1 & $12 \times 120$ & HD $207720(A 0 \mathrm{~V})$ \\
\hline
\end{tabular}

a J2000.00 coordinates from the 2MASS All-Sky Point Source Catalog.

${ }^{b}$ Number of integrations times integration time.

on 2005 July 18 UT seeing was $1.5^{\prime \prime}$, but sky transparency was otherwise fine. Sky conditions during the 2005 December 9 UT observations of 2 MASS $2148+4003$ were excellent. The data were obtained in two sets of dithered pairs, with a $300 \mathrm{~s}$ exposure obtained at each position and an air mass of 1.00 and 1.20 for 2MASS J1821+1414 and 2MASS J2148+4003, respectively. To measure telluric absorption, the A0 dwarf HD 165029 was observed for 2MASS J1821+1414 in one set (two nods) of 30 co-adds of $2 \mathrm{~s}$ each and one set of 30 co-adds of $1 \mathrm{~s}$ each, at an air mass of 1.00 and 1.01, respectively. For 2MASS J2148+4003, the A0 dwarf SAO 71693 was observed in two sets of dithered pairs with a $30 \mathrm{~s}$ exposure obtained at each position and an air mass of 1.26. Such early-type A stars are essentially featureless in this region; only the star's hydrogen Paschen line at $1.282 \mu$ m needed to be interpolated over during the telluric correction step. Calibrations consisted of neon and argon arc lamp spectra, a dark frame, and a spectrum of a flat-field lamp. We employed standard reductions using the REDSPEC package, as described in detail in McLean et al. (2003).

2MASS J2148+4003 was again obtained with NIRSPEC on 2006 August 4 UT in two more low-resolution settings covering the wavelength regions $0.94-1.15 \mu \mathrm{m}$ and $1.10-1.31 \mu \mathrm{m}$. Again, a $0.38^{\prime \prime}$ slit was used to obtain a resolving power of $R \approx 2500$. Conditions were $0.7^{\prime \prime}-1.0^{\prime \prime}$ seeing and clear skies. Data at both settings were acquired using two sets of dithered pairs with a $200 \mathrm{~s}$ exposure at each dither position. The shorter and longer wavelength regions were acquired at air masses of 1.34 and 1.57 for 2MASS J2148+4003; the A0 dwarf calibrator HD 207220 was acquired in two nod pairs having four co-adds of $15 \mathrm{~s}$ each and were taken at air masses of 1.41 and 1.48 for the short- and longwavelength pieces, respectively. Calibrations and reductions were identical to those of the earlier NIRSPEC data described above, the only difference being that the A0 star's hydrogen Paschen lines at 1.005 and $1.094 \mu \mathrm{m}$ also needed to be interpolated over during the telluric correction step.

\subsection{IRTF-SpeX Spectroscopy \\ 2.5.1. Prism Mode: $\sim 0.7-2.5 \mu \mathrm{m}$}

Near-infrared spectra for 2MASS J1821+1414 and 2MASS J2148+4003 were also obtained with SpeX (Rayner et al. 2003) on the $3.0 \mathrm{~m}$ NASA Infrared Telescope Facility (IRTF). 2MASS $\mathrm{J} 1821+1414$ was observed in prism mode on 2005 August 10 UT with scattered clouds and $\sim 0.7^{\prime \prime}$ seeing at $K$ band. The prism mode provides continuous coverage from 0.7 to $2.5 \mu \mathrm{m}$ in a single order on the $1024 \times 1024 \mathrm{InSb}$ array. Use of the $0.5^{\prime \prime}$ slit resulted in a resolving power of $R \approx 100$ in $J$ band to $\approx 300$ in $K$ band. For accurate sky subtraction, the target was observed in four nodded pairs having $120 \mathrm{~s}$ integrations per position at an air mass of 1.03 , for a total of 16 minutes of integration time. The A0 star HD 165029 was observed immediately prior to this observation at an identical air mass followed by calibration flats and argon arc lamp exposures.

2MASS J2148+4003 was observed in prism mode on 2005 September 8 UT and 2005 September 9 UT with clear conditions and $0.4^{\prime \prime}$ seeing at $H$ band on the first night and heavy cirrus with 
$0.5^{\prime \prime}-0.7^{\prime \prime}$ seeing at $H$ band on the second night. On both nights, the target was observed in four nodded pairs having $120 \mathrm{~s}$ integrations per position, for a total integration time of 16 minutes on each night. The A0 star HD 207220 was observed immediately after the science observations at a similar air mass $(\Delta z<0.1)$, followed by calibration flats and argon arc lamp exposures. For comparison to the spectrum of 2MASS J2148+4003 (see $\S 4.2$ ), 2MASSW J22443167+2043433 (hereafter 2MASS J2244+2043) was observed in prism mode on 2005 September 9 UT with the same instrumental setup as the 2MASS J2148+4003 observations. The target was observed in six nodded pairs having $120 \mathrm{~s}$ exposures per position, for a total integration time of 24 minutes. The same observation of HD 207220 that was used for the 2MASS J2148+4003 September 9 data was also used for telluric correction and flux calibration of 2MASS J2244+2043.

Standard reductions were employed using the Spextool package version 3.2 (Cushing et al. 2004; Vacca et al. 2003). The two fully reduced spectra of 2MASS J2148+4003 from 2005 September 8 UT and 2005 September 9 UT were combined into one final spectrum by interpolating the wavelength scale of the spectrum from 2005 September 9 UT onto the wavelength scale of the 2005 September 8 UT spectrum and then averaging the fluxes.

\subsubsection{Short-Wavelength Cross-Dispersed Mode: $\sim 0.9-2.4 \mu \mathrm{m}$}

2MASS J1821+1414 and 2MASS J2148+4003 were observed in short-wavelength cross-dispersed mode to provide higher resolution spectra over $0.9-2.4 \mu \mathrm{m}$ in six orders. Use of the $0.5^{\prime \prime} \mathrm{slit}$ resulted in a resolving power of $R \approx 1200$. 2MASS J1821+1414 was observed on 2005 August 10 UT at an air mass of 1.1 in six nodded pairs having $300 \mathrm{~s}$ integrations per position, for a total of 60 minutes of integration time. 2MASS J2148+4003 was observed on 2005 September 9 UT at an air mass of 1.1 in 12 nodded pairs having $120 \mathrm{~s}$ integrations per position, for a total of 48 minutes of integration time.

Observations of the A0 dwarf HD 165029 (for 2MASS J1821+ 1414) and the A0 dwarf HD 207220 (for 2MASS J2148+4003) near a similar air mass and time as the target were taken with the above observations to provide both telluric correction and flux calibration. For all observations, the instrument rotator was positioned at the parallactic angle. For instrumental calibration, internal flat-field and argon arc lamps were observed immediately after the observation of the A0 stars. Standard reductions were employed using the Spextool package version 3.2.

\subsubsection{Long-Wavelength Cross-Dispersed Mode: $\sim 1.9-4.2 \mu \mathrm{m}$}

Spectra of 2MASS J1821+1414 and 2MASS J2148+4003 were also obtained with SpeX using the long cross-dispersed mode, which provides coverage over $1.9-4.2 \mu \mathrm{m}$ in six orders. 2MASS J2148+4003 was observed on 2006 September 1 UT, 2006 September 12 UT, and 2007 November 11 UT. The night of 2006 September 1 was clear, with $0.4^{\prime \prime}$ seeing at $K$ band, the night of 2006 September 12 was mostly clear, with light cirrus, high relative humidity $\left(\sim 75 \%\right.$ ), and $0.7^{\prime \prime}$ seeing at $K$ band, and the night of 2007 November 11 was clear, with $0.9^{\prime \prime}$ seeing at $K$ band. Use of the $0.5^{\prime \prime}$ slit resulted in a resolving power of $R \approx 1500$. During the first night, the target was observed in 20 on-slit nodded pairs having $30 \mathrm{~s}$ integrations per position, totaling 20 minutes of integration. On the second night, the target was observed in three sets of 20 nodded pairs having $30 \mathrm{~s}$ integrations per position, totaling 60 minutes of integration, and on the third night, the target was observed in five sets of 20 nodded pairs having $30 \mathrm{~s}$ integrations per position, totaling 100 minutes of integration. For multiple sets on a single night, the position angle of the slit was rotated to match the parallactic angle between each set and between the science and standard observations. For telluric correction and flux calibration, the A0 dwarf HD 209932 was observed on the first two nights during a similar time and near the same air mass $(\Delta z<0.1)$ as 2MASS J2148+4003. During the third night, the A0 dwarfs HD 209932 and HD 13936 were observed at three different times to match air masses $(\Delta z<0.15)$ of the five different sets of science observations. Immediately after the A0 observations, we obtained flat-field and argon arc lamps for calibrations.

2MASS J1821+1414 was observed in long cross-dispersed mode on 2007 July 28 UT. The night was clear, with $0.8^{\prime \prime}$ seeing at $K$ band during observations. The target was observed in two sets of 20 nodded pairs having $30 \mathrm{~s}$ integrations per position, totaling 40 minutes of integration. The A0 dwarf HD 165029 was observed after the science target, followed immediately by calibration flats and argon arc lamp observations. The air mass matches between the science and standard observations were very poor $(\Delta z \sim 0.5-0.7)$, since 2MASS J1821+1414 was observed until it was setting.

Reductions were carried out using the Spextool package version 3.4. Due to the faint signal for both science targets, we first combined the spectra of each nod position for all sets, defined the apertures, wrote traces, extracted the spectra, and then combined the $\mathrm{AB}$ aperture-stacked spectra. The spectrum from each set was telluric corrected using the standard closest in time and air mass. All six orders were then merged, starting in $K^{\prime \prime}$ band (order 10) and going to progressively longer wavelengths (smaller orders) with no autoscaling employed. All sets were merged into one final spectrum, and regions of heavy telluric absorption, i.e., $\sim 2.5-3.0 \mu \mathrm{m}$, were removed. Finally, we rebinned the spectra by a factor of 3 such that 1 pixel corresponds to a single resolution element.

\subsection{Spitzer-IRS Spectroscopy}

2MASS J2148+4003 and 2MASS J1821+1414 were observed (Spitzer AORs 16201984 and 16176128) using the Short-Low (SL) module of the Infrared Spectrograph (IRS; Houck et al. 2004) onboard the Spitzer Space Telescope (Werner et al. 2004). This module covers $5.2-15.3 \mu \mathrm{m}$ at $R \approx 90$ in two orders. The observations consisted of a series of exposures taken at two different positions along each slit. The total exposure time for each target was $3904 \mathrm{~s}$. The raw data were processed with the IRS pipeline (ver. S13) at the Spitzer Science Center and were further reduced as described in Cushing et al. (2006). Briefly, the spectra were extracted with a fixed-width aperture of $6^{\prime \prime}$, and observations of $\alpha$ Lac obtained as part of the IRS calibration observations were used to remove the instrument response function and to flux-calibrate the observations of 2MASS J2148+4003 and 2MASS J1821+1414.

\section{ANALYSIS}

\subsection{Red-Optical Spectral Classification}

\subsubsection{MASS J1821+1414 (L4.5)}

We spectroscopically classify 2MASS J1821+1414 in the red-optical $(\lambda<8600 \AA)$ by comparing its continuum shape in Figure 3 to that of other known, optically classified field dwarfs: 2MASSW J1146345+223053 (L3, hereafter 2MASS J1146+2230), 2MASSW J1155009+230706 (L4, hereafter 2MASS J1155+ 2307), 2MASSW J1507476-162738 (L5, hereafter 2MASS J1507-1627; Kirkpatrick et al. 1999), and 2MASS J21321145+ 1341584 (L6, hereafter 2MASS J2132+1341; Cruz et al. 2007). Spectra of 2MASS J1507-1627 and 2MASS J2132+1341, like 2MASS J1821+1414, were obtained using Subaru-FOCAS $(R \sim 1000)$, while spectra of 2MASS J1146+2230 and 2MASS 


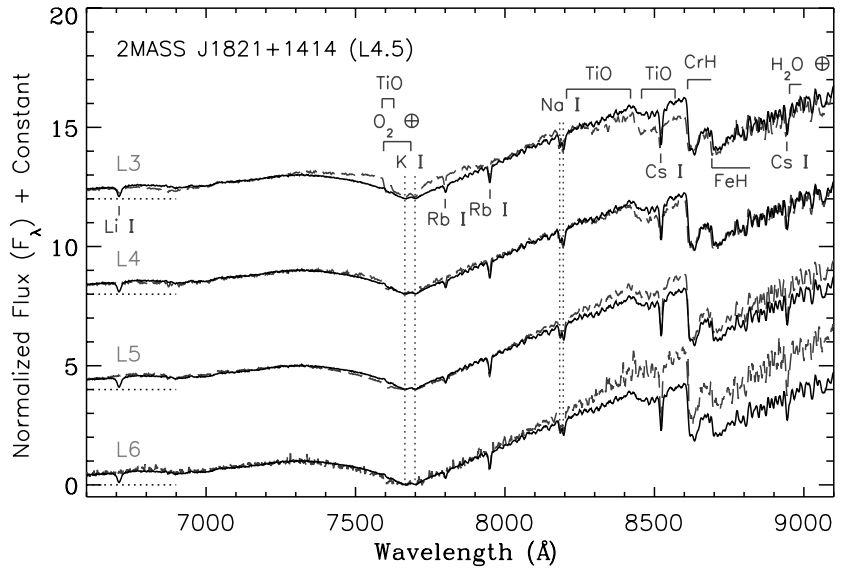

FIG. 3.-Optical spectra from Subaru-FOCAS of 2MASS J18212815+1414010 (black solid lines) compared to the typical field L dwarfs (gray dashed lines): 2MASSW J1146345+223053 (L3) and 2MASSW J1155009+230706 (L4), taken with Keck-LRIS $(R \sim 1200)$, and 2MASSW J1507476-162738 (L5) and 2MASS $\mathrm{J} 21321145+1341584$ (L6), taken with Subaru-FOCAS $(R \sim 1000)$. The spectrum of 2MASS J1821+1414 has been telluric corrected, while the spectra of the comparison L dwarfs have not. All spectra have been normalized to 1 using the median in a $20 \AA$ window centered on $7300 \AA$ and are separated along the vertical axis by a constant of 4.0 (dotted lines show zero levels) for clarity. The continuum of 2MASS J1821+1414, shortward of $8600 \AA$, appears to be intermediate between the L4 and L5 types.

J1155+2307 were obtained using Keck I-LRIS $(R \sim 1200)$. 2MASS J1821+1414 has been telluric corrected, while the comparison L dwarfs have not been telluric corrected. The best continuum fit shortward of $8600 \AA$ appears to be intermediate between the L4 and L5 dwarfs, including the strengths of the TiO band heads, as well as the $\mathrm{Rb}$ I and Cs I atomic line strengths. We therefore adopt an optical spectral classification of L4.5 for 2MASS J1821+1414.

Quite unlike the L dwarfs shown for comparison, 2MASS J1821+ 1414 shows deep Li absorption at $6708 \AA$. Using the IRAF package SPLOT, we measured an equivalent width $(\mathrm{EW}=13.9 \pm$ $0.4 \AA$ ) of this feature by taking the average of five measurements and estimating the error by the standard deviation in these measurements added in quadrature to the average of five measurements of a typical noise spike. Approximately $40 \%$ of L4-L4.5 dwarfs have a detected $\mathrm{Li}$ I absorption line (EW $>4 \AA$ ), with a median EW of 7-9 A (Kirkpatrick et al. 2008). None of the L4L4.5 dwarfs in that paper have Li EW measures as large as that seen in 2MASS J1821+1414.

\subsubsection{MASS J2148+4003 (L6)}

We determine the red-optical $(\lambda<8600 \AA)$ spectral type of 2MASS $2148+4003$ by comparing its continuum to that of known optically classified L dwarfs (Fig. 4): 2MASS J1155+2307 (L4), 2MASS J1507-1627 (L5), 2MASS J2132+1341 (L6), and DENIS-P J0205.4-1159 (L7, hereafter DENIS J0205-1159; Kirkpatrick et al. 1999). DENIS J0205-1159 was also observed with Subaru-FOCAS using a setup identical to that for 2MASS $\mathrm{J} 2148+4003$ but, like the other comparison L dwarfs, was not telluric corrected, whereas 2MASS J2148+4003 was. The continuum of 2MASS J2148+4003 shortward of $8600 \AA$ matches very well that of the L6 dwarf, including the TiO band heads, while the atomic line strengths of $\mathrm{Rb}$ I, Na I, and Cs I are slightly weaker. We therefore adopt an optical spectral type of L6 for 2MASS $\mathrm{J} 2148+4003$.

2MASS J2148+4003 also shows Li absorption in its spectrum. Using the same method described for 2MASS J1821+1414, we measure an EW of $12.1 \pm 0.6 \AA$. Nearly $70 \%$ of L6-L6.5 dwarfs have Li detected at greater than $4 \AA \mathrm{EW}$ in their spectra, and in

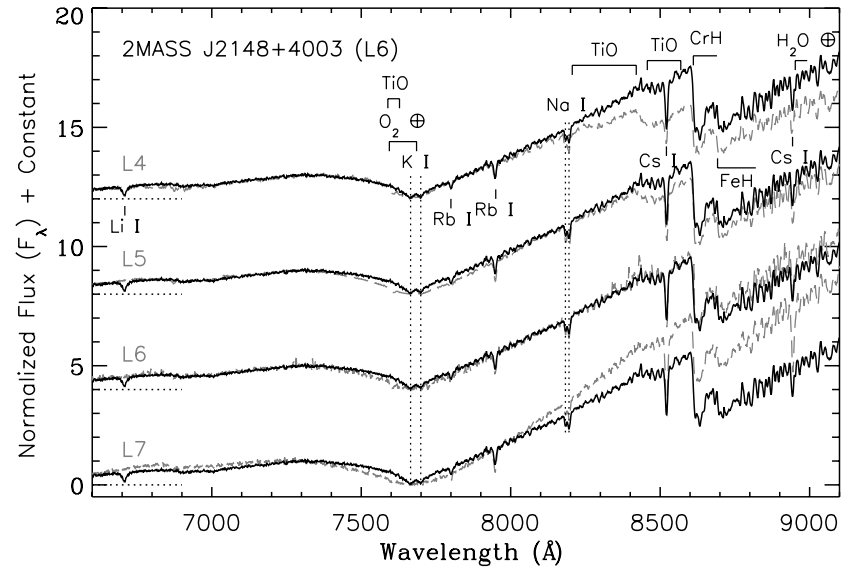

FIG. 4.- Optical spectra from Subaru-FOCAS of 2MASS J21481628+4003593 (black solid lines) compared to the typical field L dwarfs (gray dashed lines): 2MASSW J1155009+230706 (L4), taken with Keck-LRIS $(R \sim 1200)$, 2MASSW J1507476-162738 (L5), 2MASS J21321145+1341584 (L6), and DENIS-P J0205.4-1159 (L7), taken with Subaru-FOCAS $(R \sim 1000)$. The spectrum of 2MASS J2148+4003 has been telluric corrected, while the spectra of the comparison L dwarfs have not. All spectra have been normalized to 1 using the median in a $20 \AA$ window centered on $7300 \AA$ and are separated along the vertical axis by a constant of 4.0 (dotted lines show zero levels) for clarity. The continuum of 2MASS $2148+4003$, shortward of $8600 \AA$, appears to match well that of the L6 type.

these the EW ranges from 12 to $18 \AA$ (Kirkpatrick et al. 2008). 2MASS J2148+4003 appears to be a normal, Li-bearing L6 type.

\subsection{Near-Infrared Spectral Classification 3.2.1. 2MASS J1821+1414 (L5 pec)}

We compare the IRTF-SpeX cross-dispersed spectrum $(R \sim$ 1200 ) of 2 MASS J1821+1414 to a spectral sequence of normal, late-type L field dwarfs from Cushing et al. (2005) in Figure 5. The spectral types of these late-type L dwarfs are from the optical classification scheme (Kirkpatrick et al. 1999). The lowest spectral resolving power for this set is $R \approx 1200$ for 2MASSI J0825196+ 211552 (L7.5, hereafter 2MASS J0825+2115; Kirkpatrick et al. 2000) and for our object 2MASS J1821+1414. We have degraded the resolving power, $R \approx 2000$, of 2 MASS J1507-1627 and 2MASSW J1515008+484742 (L6, hereafter 2MASS J1515+ 4847; Cruz et al. 2007) to $R \approx 1200$ by convolving the spectra with a Gaussian of appropriate FWHM to reach the desired resolving power. Comparable spectral resolving powers are important when visually comparing very narrow features such as atomic lines, which change depth according to the resolving power used.

Examining Figure 5, we see that the overall spectral morphology of 2MASS J1821+1414 falls between the L6 and L7.5, but is most similar to that of the L6 type, having comparable flux levels at $J$ and $H$ bands and similar $\mathrm{H}_{2} \mathrm{O}$ absorption strengths. Nonetheless, there are notable differences between it and the L6 standard, namely, the triangular $H$ - and $K$-band continua, the stronger $\mathrm{CO}$ bands near $2.3 \mu \mathrm{m}$, the slightly shifted $K$-band peak, and the overall redder spectrum.

Since it is not uncommon for near-infrared spectral types to differ from optically derived spectral types for the same object (see $\S 3.2$ of Kirkpatrick 2005), we now focus on the $J$-band portion of the spectra of 2MASS J1821+1414 in Figure 6. We compare the Keck-NIRSPEC spectrum of 2MASS J1821+1414 to a grid of optically classified L dwarfs acquired by McLean et al. (2003) with the same telescope/instrument setup: 2MASS J215804571550098 (L4), DENIS-P J1228.2-1547 (L5, hereafter DENIS J1228-1547), 2MASSs J0850359+105716AB (L6, hereafter 2MASS J0850+1057AB), and 2MASSW J1728114+394859 (L7, 

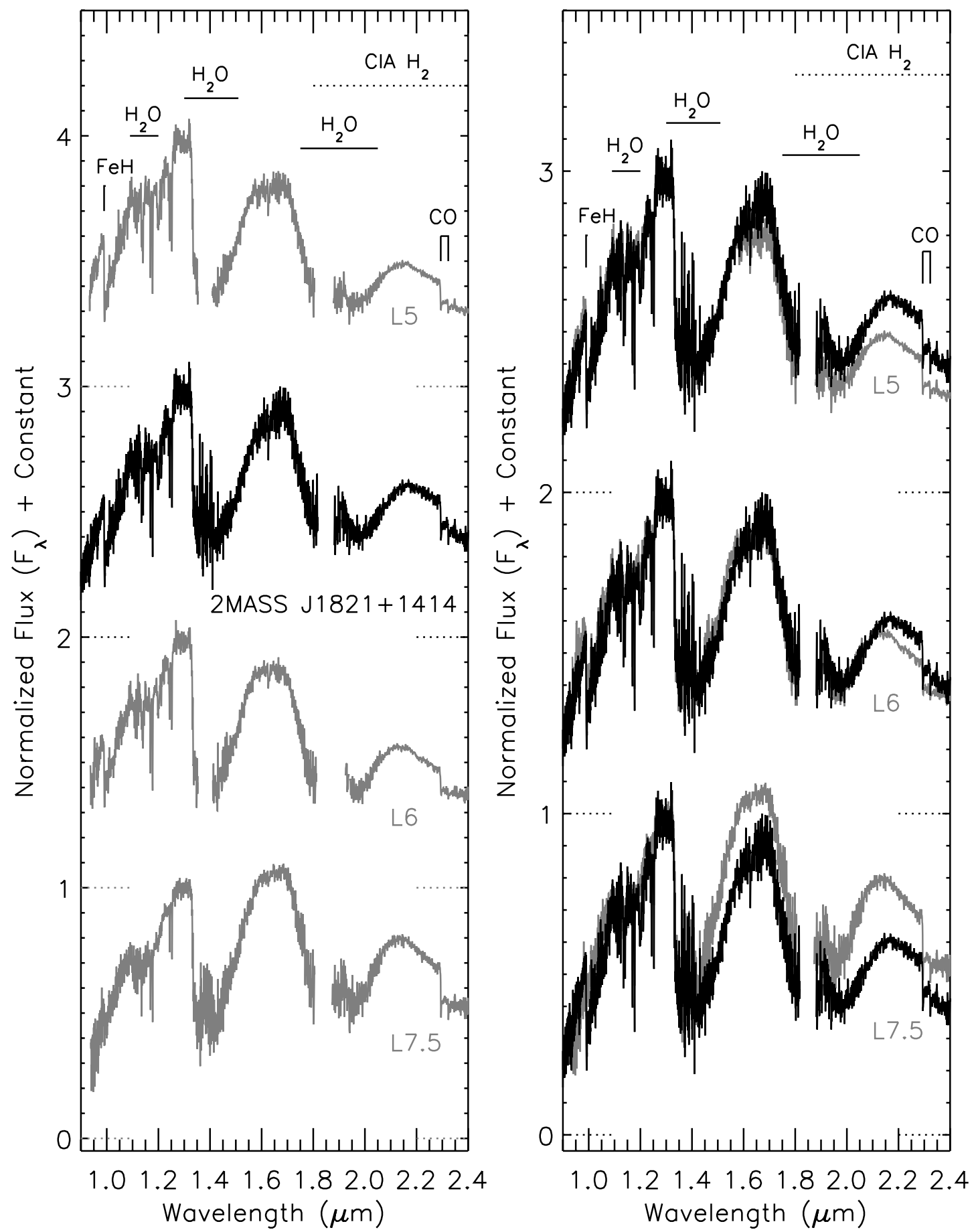

FIG. 5. - Left: Near-infrared cross-dispersed spectra from IRTF-SpeX of 2MASS J18212815+1414010 (black lines) plotted in comparison to normal, field L dwarfs (gray line): 2MASSW J1507476-162738 (L5), 2MASSW J1515008+484742 (L6), and 2MASSI J0825196+211552 (L7.5; from Cushing et al. 2005). All spectra have been normalized to 1 using the median in a $0.004 \mu \mathrm{m}$ window centered on $1.27 \mu \mathrm{m}$ and are separated along the vertical axis by a constant of 1.0 (dotted lines show zero levels) for clarity. All spectra have comparable resolving powers, $R \sim 1200$. Right: The same normalized spectra of field L dwarfs ( gray lines) overlaid with the spectrum of 2MASS J1821+1414 (black lines). While the spectrum of 2MASS J1821+1414 is slightly redder and has a somewhat peakier $H$ band, its gross spectral shape and $\mathrm{H}_{2} \mathrm{O}$ absorption strengths fit the L6 type closest.

hereafter 2MASS J1728+3948). Over this wavelength regime, the continuum from $1.26-1.32 \mu \mathrm{m}$, the strength of the $\mathrm{H}_{2} \mathrm{O}$ band redward of 1.32, and the strength of the FeH bands most closely match the L5 type. In fact, the agreement over this region is far better than that seen for any of the standards in the $H$ - or $K$-band windows.

We adopt an overall near-infrared spectral type of L5 pec for 2MASS $\mathrm{J} 1821+1414$ based on its spectral characteristics at $J$ band and peculiarities at longer wavelengths. We note that the spectrum itself deviates from a standard L5 type, as the $H$-band portion is markedly more peaked than in the standard and the overall spectrum is slightly redder. We discuss possible causes for these differences in later sections.

\subsubsection{MASS J2148+4003 (L6.5 pec)}

We follow a similar prescription of spectral analysis for 2MASS $\mathrm{J} 2148+4003$ as done above for 2MASS J1821+1414. In Figure 7, we compare the IRTF-SpeX cross-dispersed spectrum $(R \sim 1200)$ 


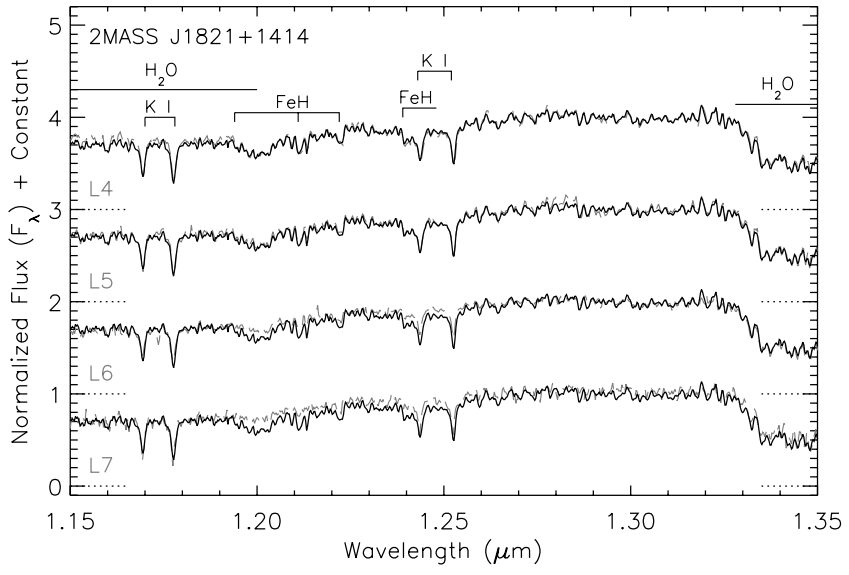

FIG. 6. - 1.14- $1.38 \mu \mathrm{m}$ Keck-NIRSPEC data $(R \sim 2000)$ of 2MASS J18212815+ 1414010 (black solid lines) overlaid on a grid of mid- to late-type L dwarfs ( gray dashed lines): 2MASS J21580457-1550098 (L4), DENIS-P J1228.2-1547 (L5), 2MASSs J0850359+105716AB (L6), and 2MASSW J1728114+394859 (L7), taken with the same telescope/instrument setup (McLean et al. 2003). All spectra have been normalized to 1 at $1.27 \mu \mathrm{m}$ using the median in a $0.004 \mu \mathrm{m}$ window. The spectra are separated vertically in integer units of 1 , with zero levels shown by dotted lines. The continuum of 2MASS J1821+1414 in this window and the $\mathrm{K}$ I and $\mathrm{FeH}$ absorption strengths match the L5 type closest.

of 2MASS J2148+4003 to a spectral sequence of normal, late-type L field dwarfs from Cushing et al. (2005): 2MASS J1515+4847 (L6), 2MASS J0825+2115 (L7.5), and DENIS-P J0255-4700 (L8; hereafter DENIS J0255-4700; Martín et al. 1999). We degrade the resolving power, $R \sim 2000$, of both 2MASS $1515+4847$ and DENIS J0255-4700 to match the resolving power, $R \sim$ 1200, of 2MASS J0825+2115 (L7.5) and 2MASS J2148+4003. Figure 7 highlights the extreme peculiarities of 2MASS J2148+ 4003: the marked redness of the NIR spectrum, the triangular $H$ - and $K$-band continua, the unusually red slope throughout the $Z, Y$, and $J$ bands, the redshifted $K$-band peak, and the large CO-band features at 2.29 and $2.32 \mu \mathrm{m}$. These two panels show that 2MASS J2148+4003 is unlike any of these late-type L dwarfs.

Now we turn to the $J$-band spectrum in Figure 8, which compares the Keck-NIRSPEC spectrum of 2MASS J2148+4003 with late-type optically classified L dwarfs taken with the same telescope/instrument setup (McLean et al. 2003): DENIS J12281547 (L5), 2MASS J0850+1057AB (L6), 2MASS J+39481728 (L7), and the very red L6.5 pec 2MASS J2244+2043. The continuum shortward of $1.26 \mu \mathrm{m}$ and the $\mathrm{K}$ I and $\mathrm{FeH}$ absorption strengths match the L6 type closest, although it should be noted that the L6 used here, 2MASSI J0103320+193536 (Kirkpatrick et al. 2000), has weaker $\mathrm{K}_{\mathrm{I}}$ lines and $\mathrm{FeH}$ band strengths than expected in the late-type L sequence. From 1.26 to $1.32 \mu \mathrm{m}$, the continuum of 2MASS J2148+4003 more closely matches the L7 type. Throughout this window, the continuum of 2 MASS J2148+ 4003 and the $\mathrm{H}_{2} \mathrm{O}$-band strengths match the L6.5 (NIR L7.5 pec) dwarf 2MASS J2244+2043 (McLean et al. 2003) very well. However, the two spectra do deviate in their finer structure; 2MASS J2244+2043 has very weak K I lines and indiscernible FeH absorption bands, presumably because it is a low-gravity object (McLean et al. 2003).

Using the results of our fits from the $J$-band windows and the overall peculiarities at $H$ and $K$ bands, we adopt an overall spectral type of L6.5 pec for 2MASS J2148+4003. We discuss possible causes for the peculiarities in $\S 4$.

\subsection{2-15.3 $\mu \mathrm{m}$ Spectroscopic Features}

Figure 9 shows the IRS spectrum of 2MASS J1821+1414 and 2MASS J2148+4003, along with the spectrum of 2MASS
J1507-1627 (L5) and 2MASSW J2224438-015852 (L4.5, hereafter 2MASS J2224-0158; Kirkpatrick et al. 2000) from Cushing et al. (2006). The spectra of 2MASS J2148+4003, 2MASS J1821+1414, and 2MASS J2224-0158 all exhibit a broad absorption feature from 9 to $11 \mu \mathrm{m}$ that is absent (or rather, weak) in the spectrum of 2MASS J1507-1627. Cushing et al. (2006) tentatively identified this feature in the spectrum of 2MASS J2224-0158 as arising from the Si-O stretching mode of the silicate grains. Burgasser et al. (2008) have also noted that 2MASS J2224-0158 (L4.5) is $\sim 0.5$ mag redder in $J-K_{S}$ than 2MASS J1507-1627 (L5), perhaps indicating a correlation between the thickness of the condensate clouds and the NIR colors of L dwarfs. Both 2MASS J2148+4003 and 2MASS J1821+ 1414 are $\sim 0.1$ and $\sim 0.5$ mag redder than the median $J-K_{S}$ values of L4-L4.5 and L6-L6.5 dwarfs, respectively, which appears to strengthen this conclusion.

\subsection{Spectrophotometric Distance Estimates}

When estimating distances, optical spectral types are preferred over NIR spectral types, because they are a better predictor of $M_{J}$, particularly for mid- to late-type L dwarfs (see Fig. 9 of Kirkpatrick 2005). We use the $M_{J}$ versus spectral type relation derived by Looper et al. (2008) to estimate the distance to 2MASS $\mathrm{J} 1821+1414$ and 2MASS J2148+4003. Using the L4.5 optical spectral type and the 2MASS-measured value of $J=13.43 \pm$ $0.03 \mathrm{mag}$ for $2 \mathrm{MASS} \mathrm{J} 1821+1414$, we estimate a distance of $9.8 \pm 1.3 \mathrm{pc}$ if it is single. The optical spectral type of L6, along with the 2MASS-measured value $J=14.15 \pm 0.04$ mag for 2MASS J2148+4003, suggests a distance of $9.9 \pm 1.3 \mathrm{pc}$ if it is single. Trigonometric parallax measurements are needed to confirm these estimates.

To place these discoveries in context, we have listed them in Table 3, along with the eight other L dwarfs known or believed to lie within $10 \mathrm{pc}$. Of these eight L dwarfs, only three have trigonometric parallax measurements. For the five objects that do not have trigonometric parallaxes, we use the Looper et al. (2008) relation to spectrophotometrically estimate their distances and list these in Table 3, as well. As with 2MASS J1821+1414 and 2MASS J2148+4003, trigonometric parallaxes are needed to confirm these distance estimates.

The discovery of these two objects has increased the number of L dwarfs within $10 \mathrm{pc}$ by $\sim 25 \%$, a substantial increase in this nearest census of the solar neighborhood. However, we are not taking into account Malmquist bias when discussing the number of objects within $10 \mathrm{pc}$. These two discoveries have arisen because the sky coverage of our proper-motion survey extends into a relatively unexplored part of the Galactic plane $\left(10^{\circ} \leq|b| \leq 15^{\circ}\right)$.

\section{DISCUSSION}

\subsection{Dusty Photospheres}

All available evidence indicates that 2MASS J1821+1414 and 2MASS J2148+4003 have unusually dusty photospheres - their unusual red slopes throughout the $Z, Y$, and $J$ bands, overall red NIR colors, weak $\mathrm{H}_{2} \mathrm{O}$ absorption, and the presence of silicate $\mathrm{ab}$ sorption at 9-11 $\mu \mathrm{m}$. The wavelength dependence of dust absorption and scattering (i.e., dust extinction) causes an object to appear redder than expected, because shorter wavelength light is attenuated and scattered more than longer wavelength light. For absorption bands that form in atmospheric layers near or below the cloud deck (e.g., the $\mathrm{H}_{2} \mathrm{O}$ absorption bands at $Y$ and $J$ band), the additional opacity provided by the condensates limits the depth in the atmosphere from which radiation can escape and thus weakens their relative strengths. The atmospheric layers above the cloud 

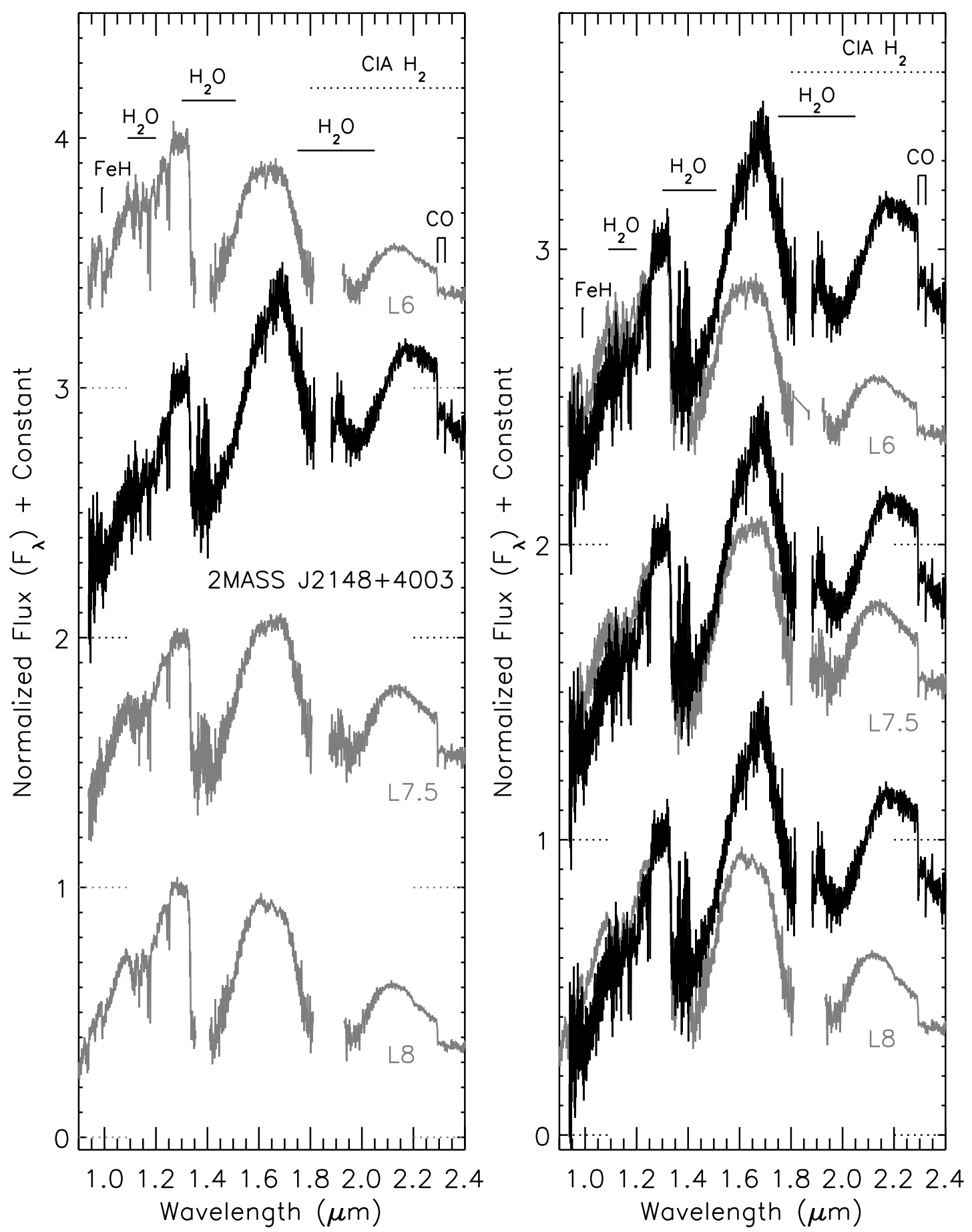

FIG. 7.-Left: IRTF-SpeX cross-dispersed spectra of 2MASS J21481628+4003593 (black lines), plotted in comparison to normal field L dwarfs (gray lines):

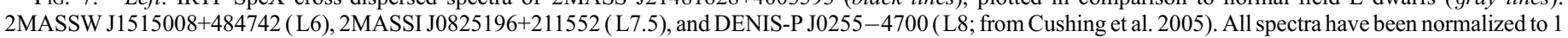
using the median in a $0.004 \mu \mathrm{m}$ window centered on $1.27 \mu \mathrm{m}$ and are separated along the vertical axis by a constant of 1.0 (zero levels are shown by dotted lines) for clarity.

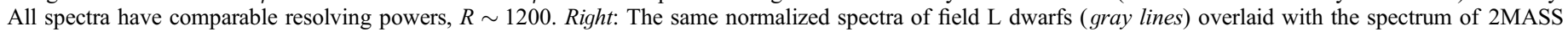
$\mathrm{J} 2148+4003$ (black lines). The spectrum of 2 MASS J2148+4003 is clearly divergent from the late-type L dwarfs, with a much redder overall spectrum, redder overall slope in the $0.9-1.3 \mu \mathrm{m}$ region, triangular $H$ - and $K$-band continua, stronger CO band absorption at 2.29 and $2.32 \mu \mathrm{m}$, and a redshifted $K$-band peak.

decks are also warmed due to the presence of the condensate clouds. Absorption lines (e.g., $\mathrm{K}_{\mathrm{I}}$ ) and bands (e.g., $\mathrm{H}_{2} \mathrm{O}$ ) that form in these atmospheric layers are therefore also weakened. Finally, the flattening seen from $9-11 \mu \mathrm{m}$ in the spectra of 2MASS J1821+1414 and 2MASS J2148+4003 is likely due to a higher bulk content of silicate dust grains in the atmosphere or from smaller grain sizes than are typical for field L dwarfs. In $\S 4.2$ we suggest two underlying physical causes for dusty photospheres.

\subsection{The Nature of Both Objects}

Below, we offer four possible explanations for the peculiar spectral features of 2MASS J1821+1414 and 2MASS J2148+ 4003 , two of which may lead to dusty photospheres.

1. Unresolved binarity.-In the case of 2 MASS J2148+4003, we can eliminate the possibility of unresolved binarity being the underlying cause for its extreme red color $\left(J-K_{s}=2.38\right)$. The 


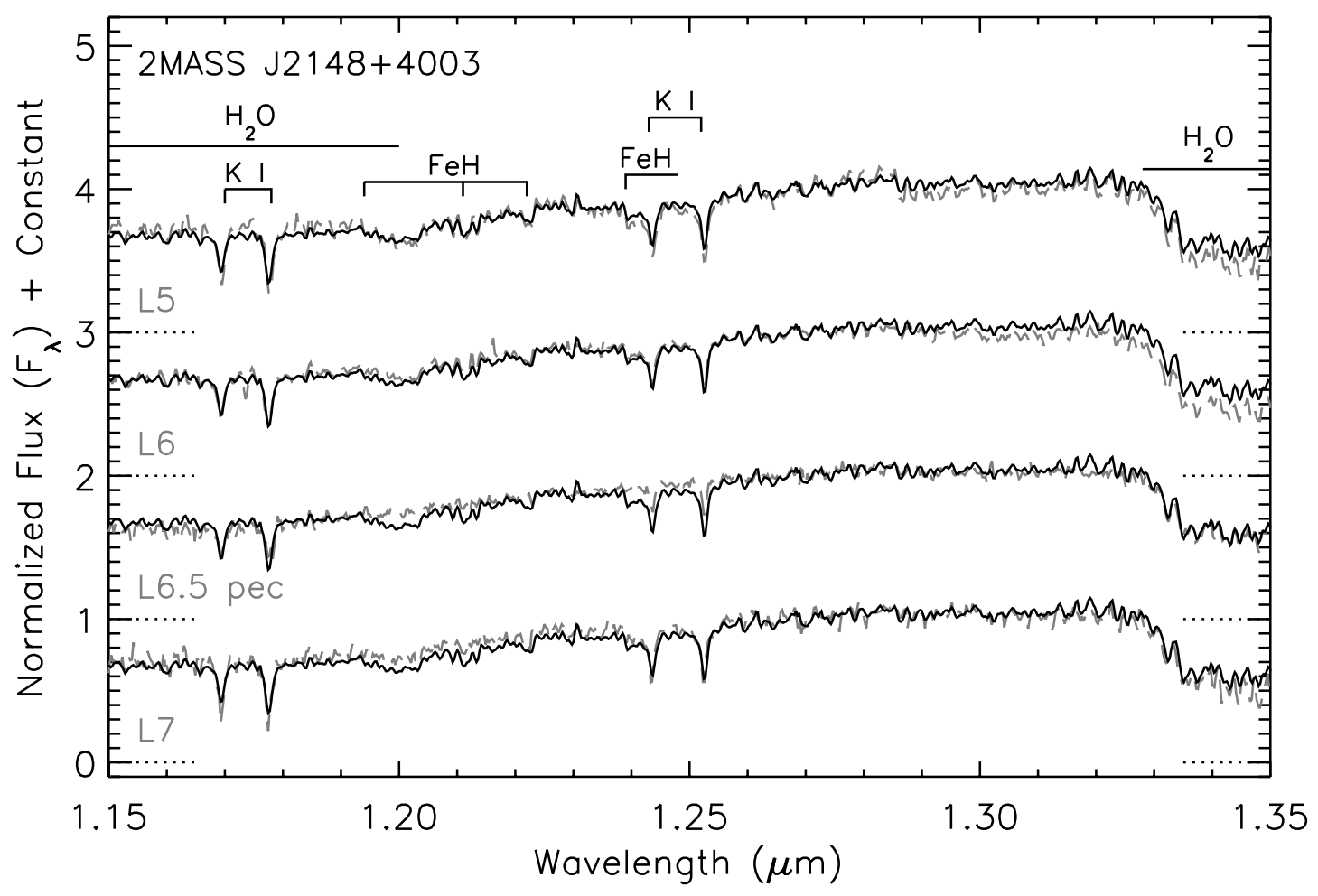

FIG. 8. - 1.14-1.38 $\mu \mathrm{m}$ Keck-NIRSPEC data $(R \sim 2000)$ of 2MASS J21481628+4003593 (black solid lines) overlaid on a grid of late-type L dwarfs (gray dashed lines): DENIS-P J1228.2 -1547 (L5), 2MASSs J0850359+105716AB (L6), 2MASSW J1728114+394859 (L7), and the very red L6.5 pec 2MASSW J2244316+204343, taken with the same telescope/instrument setup (McLean et al. 2003). All spectra have been normalized to 1 at $1.27 \mu \mathrm{m}$ using the median in a $0.004 \mu \mathrm{m}$ window. The spectra are separated vertically in integer units of 1, with zero levels shown by dotted lines. The continuum shape of 2MASS J2148+4003 is well matched to that of 2 MASS $\mathrm{J} 2244+2043$, but diverges from it in the absorption strengths of $\mathrm{K}$ I and FeH.

composite $J-K_{s}$ color of a binary results in a color intermediate between the two components, and so the composite cannot be redder than either individual member. Since 2MASS J2148+ 4003 is one of only seven L or T dwarfs with $J-K_{s}>2.3$ out of 648 objects, ${ }^{13}$ two ordinary L or T dwarf components would

${ }^{13}$ See http://dwarfarchives.org.

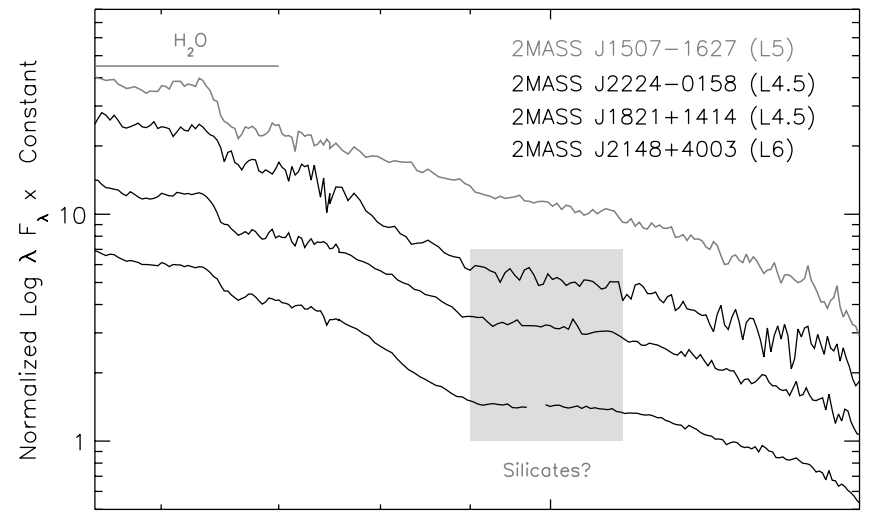

10

$\log \lambda(\mu \mathrm{m})$

FIG. 9.-Spitzer-IRS spectra $(R \sim 90)$ of 2MASSW J1507476-162738 (optical L5; top gray line), 2MASSW J2224438-015852 (optical L4.5; second black line from top), 2MASS J18212815+1414010 (optical L4.5; second black line from bottom), and 2MASS J21481628+4003593 (optical L6; bottom black line). All spectra have been normalized to 1 at $6 \mu \mathrm{m}$ using the median in a $0.4 \mu \mathrm{m}$ window. The spectra have been offset by multiplicative constants of 2. 2MASS J1507-1627 is a normal L5 dwarf, while 2MASS J2224-0158, 2MASS J1821+ 1414 , and 2MASS J2148+4003 are redder than typical L dwarfs of similar spectral type. These three objects show a flattening at $9-11 \mu \mathrm{m}$, which Cushing et al. (2006) have tentatively identified as silicate absorption. necessarily be bluer and could not combine to produce a redder composite.

Also, no known combination of $\mathrm{L}$ and $\mathrm{L}$ or of $\mathrm{L}$ and $\mathrm{T}$ dwarfs can reproduce the triangular $H$ band present in the spectra of 2MASS J1821+1414 and 2MASS J2148+4003 (see Fig. 4 of Burgasser 2007), so we must account for these features in some other way.

2. Interstellar reddening.- Interstellar material, because of its wavelength-dependent extinction properties, can cause the spectral energy distribution of a background star seen through it to be artificially reddened. However, the proper motions of these two objects alone imply that they are nearby and well within the Local Bubble (Snowden et al. 1998); hence, there is no significant amount of interstellar material expected to lie between us and these dwarfs.

3. Unusual metallicity.-Is it possible that these two objects represent unusually low or high metallicity L dwarfs? Several low-metallicity L dwarfs have already been cataloged, the most relevant examples being the sdL4 2MASS J16262034+3925190 (hereafter 2MASS J1626+3925; Burgasser 2004) and the sdL7 2MASS J05325346+8246465 (hereafter 2MASS J0532+8246; Burgasser et al. 2003). In both cases, these objects are much bluer, not redder, than field L dwarfs of the same type, the 2MASS $J-K_{s}$ values being $-0.03 \pm 0.08 \mathrm{mag}$ and $0.26 \pm 0.16 \mathrm{mag}$ for 2MASS J1626+3925 and 2MASS J0532+8246, respectively. Moreover, the near-infrared spectra of these objects do not show spectra that are sharply peaked at the long-wavelength side of $H$ band, as seen in 2MASS J1821+1414 and 2MASS J2148+4003, but instead show $H$-band spectra that are very rounded.

High metallicity may imply greater dust production, since more metals are available to condense onto grains. We examine Gaia models of high metallicity $Z=[\mathrm{Fe} / \mathrm{H}]=0.5$ versus solar metallicity $Z=0.0$ in Figure 10 . The cases shown in this figure 
TABLE 3

L Dwarfs WITHIN 10 Parsec

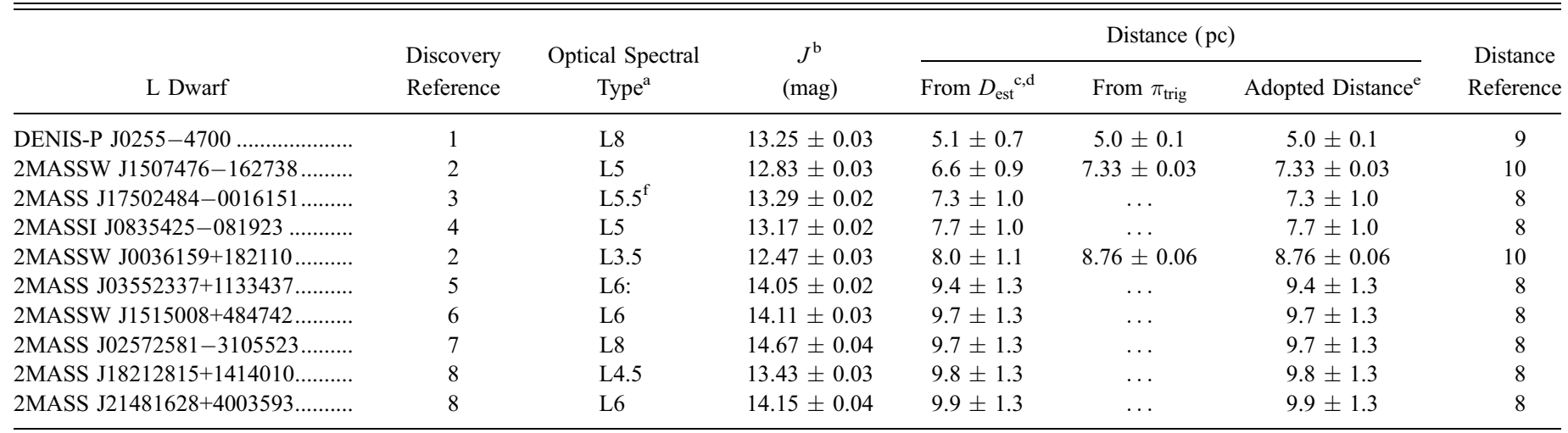

${ }^{a}$ Optical spectral types, unless otherwise noted.

b Photometry from 2MASS All-Sky Point Source Catalog.

c Spectrophotometric distance estimate; see $\S 3.4$.

${ }^{\mathrm{d}} D_{\text {est }}=10^{\left(m_{J}-M_{J}\right) / 5}, \sigma_{D}=[(\ln 10) / 5] D_{\text {est }}\left(\sigma_{m_{J}}^{2}+\sigma_{M_{J}}^{2}\right)^{1 / 2}$.

e Distance adopted from trigonometric parallax if measured.

${ }^{\mathrm{f}}$ Near-infrared spectral type.

ReferenCeS.-Discovery and distance references: (1) Martín et al. 1999; (2) Reid et al. 2000; (3) Kendall et al. 2007; (4) Cruz et al. 2003; (5) I. N. Reid et al. 2008; (6) Wilson et al. 2003; (7) J. D. Kirkpatrick et al. 2008, in preparation; (8) this paper; (9) Costa et al. 2006; (10) Dahn et al. 2002.
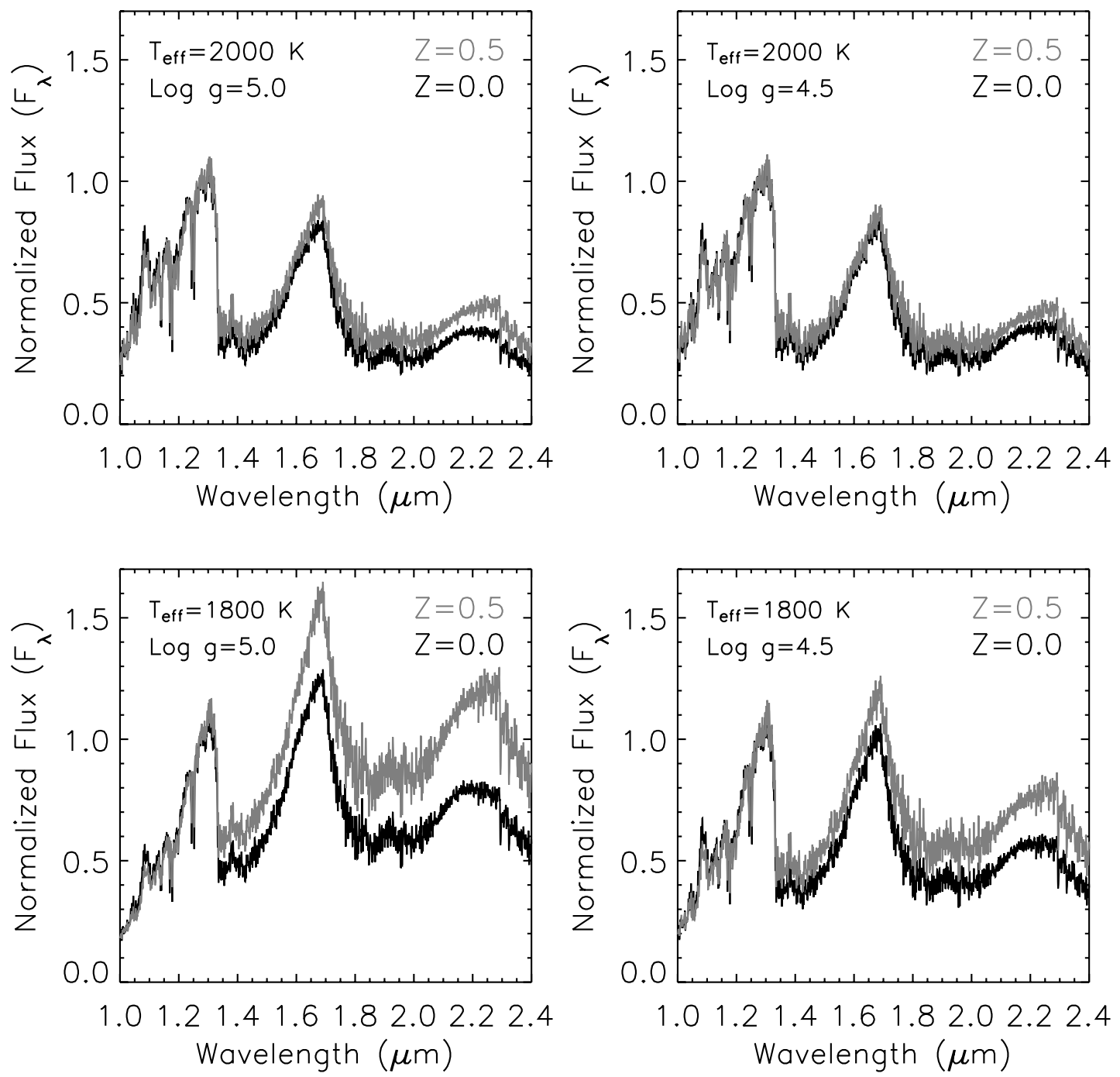

Fig. 10.-Comparison of Gaia models $(R \sim 1200)$, showing differences between solar-metallicity $Z=[\mathrm{Fe} / \mathrm{H}]=0.0$ (black lines $)$ and metal-rich $Z=0.5($ gray lines $)$ models at fixed effective temperatures and gravities. Note that the metal-rich models have comparatively redder spectra, weaker atomic lines, and weaker $\mathrm{H}_{2} \mathrm{O}$ absorption. 

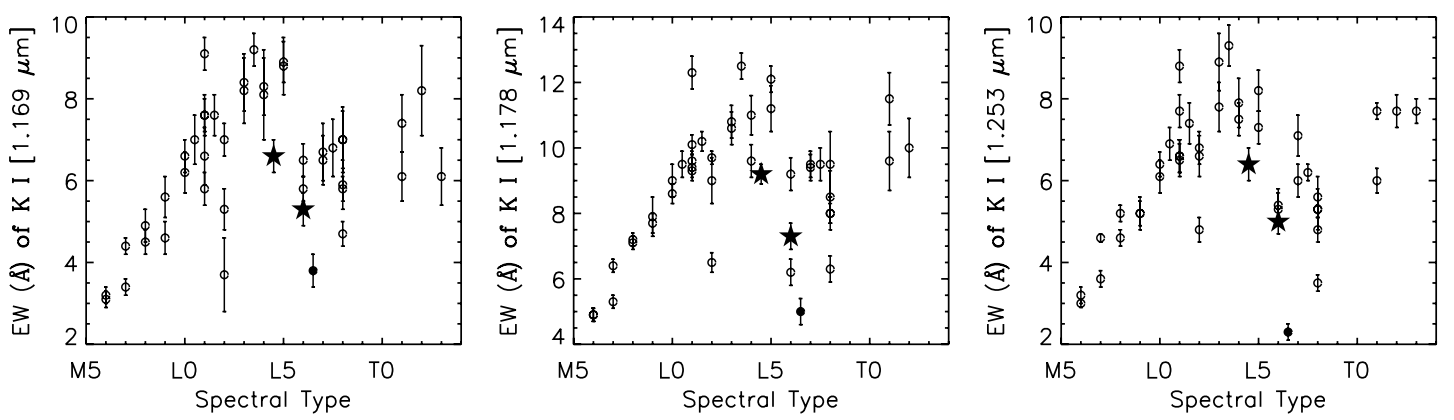

FIG. 11.-Comparison of equivalent widths for the atomic line, $\mathrm{K}$ I, at each indicated wavelength for objects with spectral types M6-T3. Field dwarfs from McLean et al. (2003) are shown as open circles, 2MASS J2244+2043, also from McLean et al. (2003) is shown as a filled circle, and 2MASS J1821+1414 and 2MASS J2148+4003 are shown as stars, with spectral types L4.5 and L6, respectively. All data are taken with Keck-NIRSPEC and a similar setup. Error bars are indicated.

are for effective temperatures and surface gravities typical for midtype $\mathrm{L}$ dwarfs. At a fixed $T_{\text {eff }}$ and surface gravity, the metalrich models have overall redder spectra, weaker $\mathrm{FeH}$ absorption, weaker $\mathrm{Na}$ I and $\mathrm{K}$ I absorption, weaker $\mathrm{H}_{2} \mathrm{O}$ absorption, and a redshifted $K$-band peak compared to the solar-metallicity models. These are the same differences noted between the two L dwarfs reported here, 2MASS J1821+1414 and 2MASS J2148+4003, and typical mid- to late-type $\mathrm{L}$ dwarfs. It is possible that these two objects could be metal-rich, causing the spectroscopic peculiarities noted here.

4. Unusual gravity.-High gravity is not favored, because it gives rise to near-infrared spectra that are bluer than normal and have spectral peaks in $H$ and $K$ bands that are rounded in appearance due to increased collision-induced absorption by $\mathrm{H}_{2}\left(\mathrm{CIA} \mathrm{H}_{2}\right)$. CIA $\mathrm{H}_{2}$ dominates the absorption at $H$ and $K$ bands (Borysow et al. 1997) and increases in strength with higher pressures.

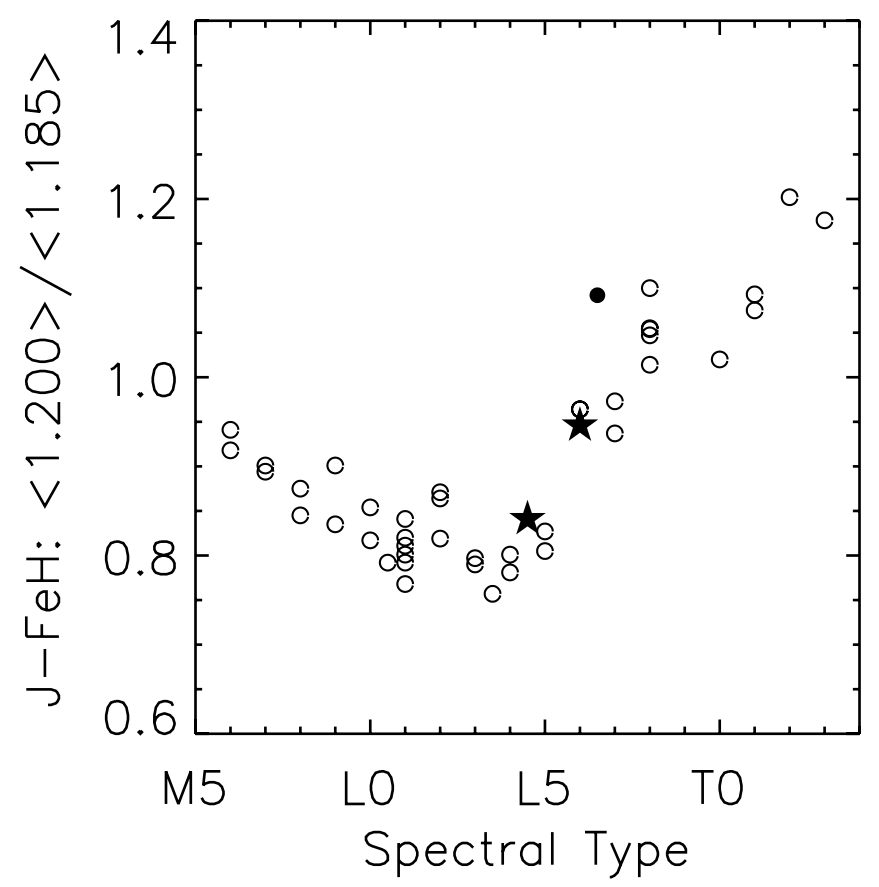

Fig. 12.-Comparison of FeH strengths using the flux ratios over a $0.004 \mu \mathrm{m}$ window centered on the FeH absorption band at $1.200 \mu \mathrm{m}$ and the pseudocontinuum at $1.185 \mu \mathrm{m}$, defined as the $J$-FeH flux ratio by McLean et al. (2003). The larger the flux ratio, the weaker the absorption. Field dwarfs from McLean et al. (2003) are shown as open circles, 2MASS J2244+2043, also from McLean et al. (2003) is shown as a filled circle, and 2MASS J1821+1414 and 2MASS J2148+ 4003 are shown as stars, with spectral types L4.5 and L6, respectively. All data are taken with Keck-NIRSPEC and a similar setup.
Low gravity, however, creates just the opposite effect. As explained in Kirkpatrick et al. (2006) low gravity results in lower atmospheric pressures, diminishing the relative importance of $\mathrm{CIA}_{2}$. With less competing opacity at $H$ band and especially at $K$ band, flux can more easily emerge at these wavelengths, making such low-gravity objects redder in NIR (JHK) colors. Lower gravity may also tend to retard the precipitation of condensates from the atmosphere, leading to thicker clouds, more condensate opacity, and redder NIR colors. Reduced $\mathrm{CIA}_{2}$ and/or increased condensate opacity are likely responsible for the triangular $H$-band spectra characteristic of low-gravity, late-type $\mathrm{M}$, and early-type L dwarfs seen both in young clusters (e.g., Orion: Lucas et al. 2001; Cha II and Rho Oph: Allers et al. 2007) and associations (e.g., Kirkpatrick et al. 2006; TWA: Looper et al. 2007a).

Both 2MASS J1821+1414 and 2MASS J2148+4003 have $H$-band spectral morphologies similar to low-gravity objects. Other gravity diagnostics, such as atomic $\mathrm{K}$ I line strengths and FeH absorption (McGovern et al. 2004), may support this hypothesis. In Figure 11, we plot the line strengths of $\mathrm{K}_{\mathrm{I}}$ at 1.169 , 1.178 , and $1.253 \mu \mathrm{m}$ for 2MASS J1821+1414 and 2MASS J2148+ 4003 in comparison to other L dwarfs, using our Keck-NIRSPEC

TABLE 4

Properties of the Two New L Dwarfs

\begin{tabular}{|c|c|c|}
\hline Parameter & 2MASS J1821+1414 & 2MASS J2148+4003 \\
\hline$J-K_{s}^{\mathrm{a}} \ldots \ldots \ldots \ldots \ldots \ldots$ & $1.78 \pm 0.04$ & $2.38 \pm 0.06$ \\
\hline$J-H^{\mathrm{a}} \ldots \ldots \ldots \ldots \ldots \ldots \ldots$ & $1.03 \pm 0.04$ & $1.37 \pm 0.06$ \\
\hline$H-K_{s}^{\mathrm{a}} \ldots \ldots \ldots \ldots \ldots \ldots$ & $0.75 \pm 0.04$ & $1.01 \pm 0.06$ \\
\hline Optical spectral type & L4.5 & L6 \\
\hline NIR spectral type.... & L5 pec & L6.5 pec \\
\hline$d_{\mathrm{est}}(\mathrm{pc}) \ldots \ldots \ldots \ldots \ldots$ & $9.8 \pm 1.3^{\mathrm{b}}(9.8 \pm 1.5)^{\mathrm{c}}$ & $9.9 \pm 1.3^{\mathrm{b}}(7.9 \pm 1.2)^{\mathrm{c}}$ \\
\hline$v_{\tan }\left(\mathrm{km} \mathrm{s}^{-1}\right) \ldots \ldots \ldots \ldots$ & $9.8 \pm 1.4$ & $62.4 \pm 3.3(49.8 \pm 3.1)^{\mathrm{d}}$ \\
\hline
\end{tabular}

Bolometric Measurements/Estimates

\begin{tabular}{lcc}
\hline $\log \left(L_{\text {bol }} / L_{\odot}\right)^{\mathrm{e}} \ldots \ldots \ldots \ldots . .$. & $-4.12 \pm 0.12$ & $-4.07 \pm 0.12$ \\
$m_{\text {bol }}(\mathrm{mag}) \ldots \ldots \ldots \ldots . .$. & $15.01 \pm 0.01$ & $14.91 \pm 0.01$ \\
$M_{\text {bol }}(\mathrm{mag}) \ldots \ldots \ldots \ldots \ldots$. & $15.05 \pm 0.29$ & $14.93 \pm 0.29$ \\
$f_{\text {bol }}\left({ }^{\mathrm{W}}{ }^{-2}\right) \ldots \ldots \ldots .$. & $(2.51 \pm 0.03) \mathrm{E}-14$ & $(2.77 \pm 0.04) \mathrm{E}-14$ \\
\hline
\end{tabular}

${ }^{a}$ Colors are computed from the values listed in the 2MASS Point Source Catalog; see Table 1.

b Distance estimate is from the optical spectral type vs. $M_{J}$ relation derived by Looper et al. (2008).

${ }^{\mathrm{c}}$ Distance estimate is from the optical spectral type vs. $M_{K_{s}}$ relation derived by Looper et al. (2008).

d Based on a distance estimate of $7.9 \mathrm{pc}$.

${ }^{\text {e }} \log \left(L_{\text {bol }} / L_{\odot}\right)=\left(M_{\text {bol } \odot}-M_{\text {bol }}\right)(2 / 5)$, where $M_{\text {bol } \odot}=+4.76$.

${ }^{\mathrm{f}}$ Values for $m_{\mathrm{bol}}$ and $f_{\mathrm{bol}}$ are independent of distance. 


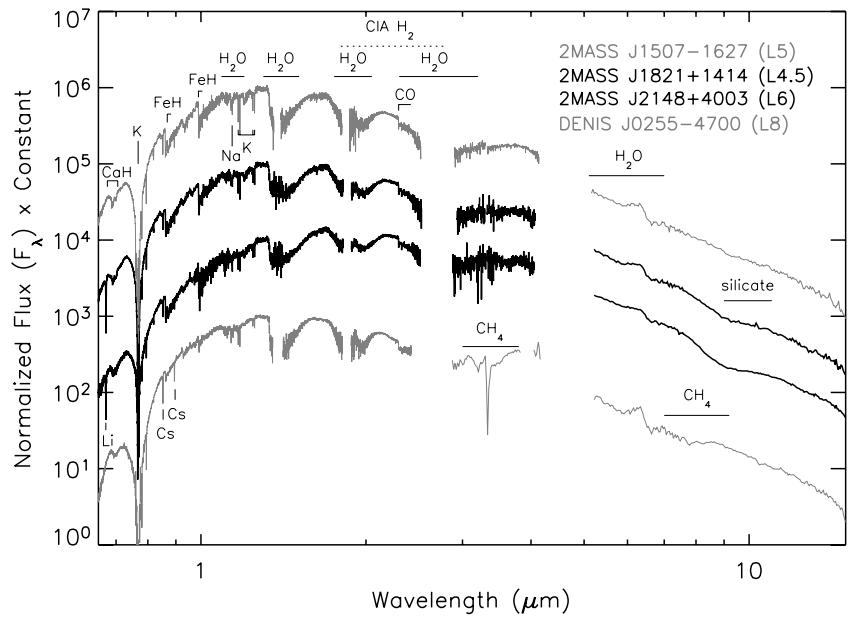

FIG. 13.-Combined optical (Subaru-FOCAS), near-infrared (IRTF-SpeX), and mid-infrared (Spitzer-IRS) spectra of 2MASSW J1507476-162738 (optical L5; top gray line), 2MASS J18212815+1414010 (optical L4.5; second black line from top), 2MASS J21481628+4003593 (optical L6; second black line from bottom), and DENIS-P J0255-4700 (optical L8; bottom gray line). All spectra have been normalized to 1.0 using the median in a $0.04 \mu \mathrm{m}$ window centered on $1.27 \mu \mathrm{m}$ and are multiplied by constants to clearly separate the spectra. Important molecular and atomic features are labeled throughout this regime.

data. The $\mathrm{K}$ I line strengths of 2MASS J1821+1414 and 2MASS $\mathrm{J} 2148+4003$ are weaker than those of other L dwarfs of similar spectral type, but are not as extreme outliers as the possibly lowgravity L dwarf 2MASS J2244+2043. Similarly, the $J$-FeH flux ratio, defined by McLean et al. (2003), shows somewhat weaker FeH absorption in 2MASS J1821+1414 and FeH absorption in 2MASS J2148+4003 comparable to normal L dwarfs of similar spectral types (see Fig. 12). 2MASS J2244+2043, on the other hand, shows markedly weaker FeH absorption. The weaker atomic lines seen in the spectra of 2MASS J1821+1414 and 2MASS J2148+4003 could also be a consequence of the higher opacity caused by thick dust clouds in their atmospheres, as explained in $\S 4.1$.

Based on their spectrophotometric distance estimates of $10 \mathrm{pc}$, we calculate the tangential velocities for both objects (see Table 4). 2MASS J1821+1414 has a low $v_{\text {tan }}$ of $\sim 10 \mathrm{~km} \mathrm{~s}^{-1}$, suggesting that it could indeed be young. However, 2MASS J2148+4003 has a large $v_{\tan }$ of $\sim 62 \mathrm{~km} \mathrm{~s}^{-1}$, well above the median value for L dwarfs of $24.5 \mathrm{~km} \mathrm{~s}^{-1}$ (Vrba et al. 2004). This large tangential velocity suggests that 2 MASS J $2148+4003$ is not young.

\subsection{Bolometric Luminosity Measures}

In Figure 13, we show the nearly complete $0.6-15.3 \mu \mathrm{m}$ spectroscopy of 2MASS J1821+1414 and 2MASS J2148+4003 compared to a typical L5 and L8 dwarf. To create a spectral energy distribution (SED), we interpolated between the Subaru-FOCAS, IRTF-SpeX prism, IRTF-SpeX long cross-dispersed, and SpitzerIRS spectra. All data but the Spitzer-IRS spectrum were fluxcalibrated with appropriate photometric measurements before being stitched together. No photometric measurements of 2MASS $\mathrm{J} 1821+1414$ and 2MASS J2148+4003 in the $5.2-15.3 \mu \mathrm{m}$ range were taken, but Cushing et al. (2006) have shown that the absolute flux calibrations are good to $\sim 3 \%$. Since no data were available shortward of $\sim 0.6 \mu \mathrm{m}$, we interpolated between the flux level at $0.6 \mu \mathrm{m}$ and a flux level of zero at $0 \mu \mathrm{m}$. Longward of $14.5 \mu \mathrm{m}$, we connected a Raleigh-Jeans tail. The bolometric luminosity was then computed by integrating underneath this SED and assuming distances of $9.8 \pm 1.3 \mathrm{pc}$ and $9.9 \pm 1.3 \mathrm{pc}$ for 2MASS J1821+1414 and 2MASS J2148+4003, respectively (see Table 4).

Note that, although 2MASS J2148+4003 has a distance estimate nearly identical to 2MASS J1821+1414 $(\sim 10 \mathrm{pc})$ and has a later spectral type, its apparent bolometric magnitude is brighter than 2MASS J1821+1414, indicating that it is actually closer than 2MASS J1821+1414. This is not surprising, if this object does have a much dustier atmosphere, causing an abnormally faint $J$-band flux compared to typical L6 dwarfs. Since the spectrophotometric distance estimate we used is based on the $J$ band, this would overestimate the distance to this object. In comparison, the $M_{K_{s}}$ versus spectral type relation derived by Looper et al. (2008) yields an identical distance estimate of $9.8 \pm 1.5 \mathrm{pc}$ for 2MASS J1821+1414, but a closer distance estimate of $7.9 \pm$ $1.2 \mathrm{pc}$ for $2 \mathrm{MASS} \mathrm{J} 2148+4003$.

\section{CONCLUSIONS}

We have presented spectral classification and distance estimates for two nearby peculiar L dwarfs identified in our $\sim 4700 \mathrm{deg}^{2}$ 2MASS proper-motion search. With optical spectral types of L4.5 and L6, we estimate distances of $\sim 10$ pc for both 2MASS J1821+1414 and 2MASS J2148+4003, although 2MASS J2148+ 4003 may actually be closer. Both objects have overall red spectral energy distributions, weak $\mathrm{H}_{2} \mathrm{O}$ bands, slightly triangular $H$-band continua, and silicate absorption at $9-11 \mu \mathrm{m}$. These features are likely due to unusually thick dust clouds in their atmospheres. While the underlying cause for this dust is uncertain, we suggest that it may arise from a metal-rich atmosphere or a lower surface gravity, the latter implying a young age. The former cause is more likely in the case of 2MASS J2148+4003, whose large $v_{\text {tan }}$ indicates that it is not a young source. Further follow-up is needed to distinguish between these two scenarios and to identify clear diagnostics for characterizing the detailed physical properties of other L dwarfs in the vicinity of the Sun.

We thank our referee, Nigel Hambly, for a timely and helpful report. We would like to thank our Keck observing assistants Steven Magee and Julie Rivera and our IRTF observing assistants Paul Sears and Dave Griep for providing expert operation of the telescopes during our runs. We thank our instrument scientists Jim Lyke and Grant Hill at Keck and Bobby Bus and John Rayner at IRTF for their expertise in running the spectrographs, imagers, and reduction software packages. We thank Takashi Hattori for providing instrument support at Subaru. We would like to thank John Stauffer and Maria Morales-Calderon for giving us some telescope time in exchange for instrument expertise on 2005 December 9 (UT) at Keck II. D. L. L. thanks John Rayner for advising her on part of this project and Dave Sanders for financial support. We are grateful to Lee Rottler for assistance with setting up the REDSPEC package at IPAC. This paper uses data from the Brown Dwarf Spectroscopic Survey Archive (http://www.astro.ucla.edu/ mclean/BDSSarchive), the IRTF Spectral Library (http://irtfweb.ifa.hawaii.edu/ spex/ spexlibrary/IRTFlibrary.html), the SpeX Prism Library (http:// web.mit.edu/ajb/www/browndwarfs/spexprism/index.html), and http://DwarfArchives.org. D. L. L. would like to thank the Northrop Grumman Corporation for a small grant to IPAC, which paid for partial salary support during the search phase of this project. This publication also makes use of data products from the Two Micron All Sky Survey (2MASS), which is a joint project of the University of Massachusetts and the Infrared Processing and Analysis Center/California Institute of Technology, funded by the 
National Aeronautics and Space Administration and the National Science Foundation. D. L. L. is a guest user of the Canadian Astronomy Data Centre, which is operated by the Herzberg Institute of Astrophysics, National Research Council of Canada. This research has also made use of the NASA/IPAC Infrared Science Archive (IRSA), which is operated by the Jet Propulsion Laboratory, California Institute of Technology, under contract with the National Aeronautics and Space Administration. The $2006 \mathrm{Au}-$ gust 4 UT NIRSPEC data were obtained at the W. M. Keck Observatory from telescope time allocated to the National Aeronautics and Space Administration through the agency's scientific partnership with the California Institute of Technology and the University of California. The Observatory was made possible by the generous financial support of the W. M. Keck Foundation. As all spectroscopic and imaging follow-up data were obtained from the summit of Mauna Kea, the authors wish to recognize and acknowledge the very significant cultural role and reverence that this mountaintop has always had within the indigenous Hawaiian community. We are most fortunate to have the opportunity to conduct observations there.
Allers, K. N., et al. 2007, ApJ, 657, 511

Barnard, E. E. 1916, AJ, 29, 181

Borysow, A., Jorgensen, U. G., \& Zheng, C. 1997, A\&A, 324, 185

Burgasser, A. J. 2004, ApJ, 614, L73

. 2007, ApJ, 659, 655

Burgasser, A. J., Looper, D. L., Kirkpatrick, J. D., Cruz, K. L., \& Swift, B. J. 2008, ApJ, 674, 451

Burgasser, A. J., Looper, D. L., Kirkpatrick, J. D., \& Liu, M. C. 2007, ApJ, 658,557

Burgasser, A. J., et al. 2003, ApJ, 592, 1186

Costa, Edgardo, Méndez, R. A., Jao, W.-C., Henry, T. J., Subasavage, J. P., \& Ianna, P. A. 2006, AJ, 132, 1234

Cruz, K. L., Reid, I. N., Liebert, J., Kirkpatrick, J. D., \& Lowrance, P. J. 2003, AJ, 126, 2421

Cruz, K. L., et al. 2007, AJ, 133, 439

Cushing, M. C., Rayner, J. T., \& Vacca, W. D. 2005, ApJ, 623, 1115

Cushing, M. C., Vacca, W. D., \& Rayner, J. T. 2004, PASP, 116, 362

Cushing, M. C., et al. 2006, ApJ, 648, 614

Dahn, C. C., et al. 2002, AJ, 124, 1170

Deacon, N. R., \& Hambly, N. C. 2007, A\&A, 468, 163

Deacon, N. R., Hambly, N. C., \& Cooke, J. A. 2005, A\&A, 435, 363

Epchtein, N., et al. 1997, Messenger, 87, 27

Finch, C. T., Henry, T. J., Subasavage, J. P., Jao, W.-C., \& Hambly, N. C. 2007, AJ, 133, 2898

Giclas, H. L., Burnham, R., \& Thomas, N. G. 1978, Lowell Obs. Bull., 8, 89

Hamuy, M., Suntzeff, N. B., Heathcote, S. R., Walker, A. R., Gigoux, P., \& Phillips, M. M. 1994, PASP, 106, 566

Houck, J. R., et al. 2004, ApJS, 154, 18

Innes, R. T. A. 1915, Union Obs. Circ., 30

Kashikawa, N., et al. 2002, PASJ, 54, 819

Kendall, T. R., Jones, H. R. A., Pinfield, D. J., Pokorny, R. S., Folkes, S., Weights, D., Jenkins, J. S., \& Mauron, N. 2007, MNRAS, 374, 445

Kirkpatrick, J. D. 2005, ARA\&A, 43, 195

Kirkpatrick, J. D., Barman, T. S., Burgasser, A. J., McGovern, M. R., McLean, I. S., Tinney, C. G., \& Lowrance, P. J. 2006, ApJ, 639, 1120

Kirkpatrick, J. D., et al. 1999, ApJ, 519, 802

. 2000, AJ, 120, 447

. 2008, ApJ, submitted

Lépine, S. 2008, AJ, 135, 2177

Lépine, S., Shara, M. M., \& Rich, R. M. 2002, AJ, 124, 1190

Lepine, S., Shara, M. M., Rich, R. M., Wittenberg, A., Halmo, M., \& Bongiorno, B. 2008, in IAU Symp. 248, A Giant Step: From Milli- to Micro-Arcsecond Astrometry (New York: IAU), 74
REFERENCES

Looper, D. L., Burgasser, A. J., Kirkpatrick, J. D., \& Swift, B. J. 2007a, ApJ, 669, L97

Looper, D. L., Gelino, C. R., Burgasser, A. J., \& Kirkpatrick, J. D. 2008, ApJ, submitted (arXiv:0803.0544)

Looper, D. L., Kirkpatrick, J. D., \& Burgasser, A. J. 2007b, AJ, 134, 1162

Lucas, P. W., Roche, P. F., Allard, F., \& Hauschildt, P. H. 2001, MNRAS, 326, 695

Luyten, W. 1979, New Luyten Catalogue of Stars with Proper Motions Larger than Two Tenths of an Arcsecond, Vol. I-II (Minneapolis: Univ. Minnesota) - 1980, New Luyten Catalogue of Stars with Proper Motions Larger than Two Tenths of an Arcsecond, Vol. III-IV (Minneapolis: Univ. Minnesota)

Martín, E. L., Delfosse, X., Basri, G., Goldman, B., Forveille, T., \& Zapatero Osorio, M. R. 1999, AJ, 118, 2466

Massey, P., \& Gronwall, C. 1990, ApJ, 358, 344

McGovern, M. R., Kirkpatrick, J. D., McLean, I. S., Burgasser, A. J., Prato, L., \& Lowrance, P. J. 2004, ApJ, 600, 1020

McLean, I. S., Graham, J. R., Becklin, E. E., Figer, D. F., Larkin, J. E., Levenson, N. A., \& Teplitz, H. I. 2000, Proc. SPIE, 4008, 1048

McLean, I. S., McGovern, M. R., Burgasser, A. J., Kirkpatrick, J. D., Prato, L., \& Kim, S. S. 2003, ApJ, 596, 561

McLean, I. S., et al. 1998, Proc. SPIE, 3354, 566

Monet, D. G., et al. 2003, AJ, 125, 984

Pokorny, R. S., Jones, H. R. A., Hambly, N. C., \& Pinfield, D. J. 2004, A\&A, 421,763

Rayner, J. T., Toomey, D. W., Onaka, P. M., Denault, A. J., Stahlberger, W. E., Vacca, W. D., Cushing, M. C., \& Wang, S. 2003, PASP, 115, 362

Reid, I. N., Cruz, K. L., Kirkpatrick, J. D., Allen, P. R., Mungall, F., Liebert, J., Lowrance, P., \& Sweet, A. 2008, AJ, 136, 1290

Reid, I. N., Kirkpatrick, J. D., Gizis, J. E., Dahn, C. C., Monet, D. G., Williams, R. J., Liebert, J., \& Burgasser, A. J. 2000, AJ, 119, 369

Ross, F. E. 1939, AJ, 48, 163

Skrutskie, M. F., et al. 2006, AJ, 131, 1163

Snowden, S. L., Egger, R., Finkbeiner, D. P., Freyberg, M. J., \& Plucinsky, P. P. 1998, ApJ, 493, 715

Vacca, W. D., Cushing, M. C., \& Rayner, J. T. 2003, PASP, 115, 389

Vrba, F. J., Henden, A. A., Luginbuhl, C. B., Guetter, H. H., \& Munn, J. A. 2004, AJ, 127, 2948

Werner, M. W., et al. 2004, ApJS, 154, 1

Wilson, J. C., Miller, N. A., Gizis, J. E., Skrutskie, M. F., Houck, J. R., Kirkpatrick, J. D., Burgasser, A. J., \& Monet, D. G. 2003, in IAU Symp. 211, Brown Dwarfs, ed. E. Martín (San Francisco: ASP), 197

Wolf, M. 1918, Astron. Nachr., 206, 237

York, D. G., et al. 2000, AJ, 120, 1579 